\title{
New Upper Bounds for Equiangular Lines by Pillar Decomposition
}

\author{
Emily J. King \\ Faculty 3: Mathematics / Computer Sciences \\ University of Bremen, 28359 Bremen, Germany \\ Xiaoxian Tang* \\ Department of Mathematics \\ Texas A\&M University, College Station, TX 77843-3368, USA
}

\begin{abstract}
We derive a procedure for computing an upper bound on the number of equiangular lines in various Euclidean vector spaces by generalizing the classical pillar decomposition developed by (Lemmens and Seidel, 1973); namely, we use linear algebra and combinatorial arguments to bound the number of vectors within an equiangular set which have inner products of certain signs with a negative clique. After projection and rescaling, such sets are also certain spherical two-distance sets, and semidefinite programming techniques may be used to bound the size. Applying our method, we prove new relative bounds for the angle $\arccos (1 / 5)$. Experiments show that our relative bounds for all possible angles are considerably less than the known semidefinite programming bounds for a range of larger dimensions. Our computational results also show an explicit bound on the size of a set of equiangular lines in $\mathbb{R}^{r}$ regardless of angle, which is strictly less than the well-known Gerzon's bound if $r+2$ is not a square of an odd number:

$$
\begin{cases}\frac{4 r(m+1)(m+2)}{(2 m+3)^{2}-r} & r=44,45,46,76,77,78,117,118,166,222,286,358 \\ \frac{\left((2 m+1)^{2}-2\right)\left((2 m+1)^{2}-1\right)}{2} & \text { other } r \text { between } 44 \text { and } 400,\end{cases}
$$
\end{abstract}

where $m$ is the largest positive integer such that $(2 m+1)^{2} \leq r+2$.

Keywords: equiangular lines, pillar decomposition, Lemmens and Seidel's conjecture, semidefinite programming, spherical two-distance sets

2010 MSC: 05B20, 05B40

\section{Introduction}

In this paper, we are concerned with the maximum number of equiangular lines in Euclidean vector spaces:

\footnotetext{
${ }^{*}$ Corresponding author

Email addresses: king@math.uni-bremen.de (Emily J. King), xiaoxian@math.tamu.edu (Xiaoxian Tang)

$U R L$ : sites.google.com/site/rootclassification (Xiaoxian Tang)
} 
Problem Statement. For a given integer $r$ with $r \geq 2$, what is the maximum number of distinct lines in $r$-dimensional Euclidean space $\mathbb{R}^{r}$ such that the angle between each pair of lines equals $\arccos (\alpha)$ for some $0<\alpha<1$ ?

By selecting a unit vector in each line in a set of equiangular lines, we can formally define it as an equiangular set of unit vectors.

Definition 1. We say $X=\left\{x_{1}, \ldots, x_{s}\right\} \subset \mathbb{R}^{r}$ is a set of equiangular lines (or, simply equiangular) if for some $0<\alpha<1$,

$$
\left\langle x_{i}, x_{j}\right\rangle= \begin{cases}1, & i=j \\ \pm \alpha, & i \neq j .\end{cases}
$$

By slight abuse of terminology, we will say that vectors which satisfy the equality (1) are equiangular with angle $\alpha$, even though the actual angle is $\arccos (\alpha)$. The ambiguity of the sign of the inner product is due to the choice of a unit vector in each line.

Fix the dimension $r(r \geq 2)$ and the angle $0<\alpha<1$. We define $s_{\alpha}(r)$ to be the maximum cardinality of an equiangular set in $\mathbb{R}^{r}$ with angle $\alpha$. Further $s(r)$ is defined to be the maximum cardinality of any equiangular set in $\mathbb{R}^{r}$, that is,

$$
s(r) \triangleq \max _{\alpha>0} s_{\alpha}(r) .
$$

Now the problem statement can be precisely rewritten:

Problem Statement'. For a given integer $r$ with $r \geq 2$, what is $s(r)$ ?

Motivation. The problem of finding the maximum number of equiangular lines has been studied for at least 70 years (Haantjes, 1948). Equivalence classes of sets of equiangular lines are equivalent to so-called two-graphs (not be be confused with 2-graphs) and are intricately connected with many problems in algebraic graph theory (Godsil and Royle, 2001). They are also equivalent to spherical codes with particular angle sets (Delsarte et al., 1977). In a seminal paper (Conway et al., 1996), the authors credit a post to a newsgroup in 1992 from an oncologist named Julian Rosenman for their interest in the field. The post asked the best way to separate laser beams going through a particular tumor, which can be thought of as asking for in-some-sense optimal packings of lines in $\mathbb{R}^{3}$. From the point of view of applications, in certain extremal cases, equiangular sets have further desirable properties with respect to data analysis and coding theory (Conway et al., 1996; Strohmer and Heath, 2003). These special sets are called equiangular tight frames (ETF). An ETF is an equiangular set $\left\{x_{1}, \ldots, x_{s}\right\} \subset \mathbb{R}^{r}$ such that for any $y \in \mathbb{R}^{r}$, the following Parseval-like equality holds

$$
\frac{s}{r}\langle y, y\rangle=\sum_{j=1}^{s}\left\langle y, x_{j}\right\rangle^{2}
$$

ETFs solve a packing problem in Grassmannian space Benedetto and Kolesar, 2006; Conwav et al., 1996; Dhillon et al., 2008), are known to be optimally robust to erasures (Strohmer and Heath, 2003; Bodmann, 2007), and further have optimal coherence which is related to the appropriateness of using a set of vectors for sparse coding (Donoho and Elad, 2003; Bruckstein et al., 2009).

The theory of equiangular lines and frames is related to linear algebra (e.g., existence of certain matrices (Lemmens and Seidel, 1973; Van Lint and Seidel,, 1966; Sustik et al., 2007)), combinatorial 
group theory (e.g., difference sets (Xia et al., 2005; Ding and Feng, 2007)), geometry (e.g., regular spherical polytopes (Coxeter, 1963)), graph theory (e.g., [regular] two-graphs and strongly regular graphs (Holmes and Paulsen, 2004; Van Lint and Seidel, 1966) ), combinatorial designs (e.g., Steiner systems (Fickus et al., 2012)), Jacobi polynomial expansions ((Delsarte et al., 1977)), and more.

Table 1 presents the currently known $s(r)$ for dimensions $2 \leq r \leq 43$. An attractive direction

Table 1: Maximum number of equiangular lines for small dimensions Lemmens and Seidel, 1973; Waldron, 2009; Barg and Yu, 2014; Greaves et al., 2016; Yu, 2015; Azariia and Marc, 2016; Szöllösi, 2017; Greaves, 2018; Greaves and Yatsyna, 2018)

\begin{tabular}{c||cccccc}
$r$ & 2 & 3 & 5 & 6 & $7-13$ & 14 \\
$s(r)$ & 3 & 6 & 10 & 16 & 28 & $28-29$ \\
\hline \hline$r$ & 15 & 16 & 17 & 18 & 19 & 20 \\
$s(r)$ & 36 & $40-41$ & $48-49$ & $54-60$ & $72-75$ & $90-95$ \\
\hline \hline$r$ & 21 & 22 & $23-41$ & 42 & 43 & \\
$s(r)$ & 126 & 176 & 276 & $276-288$ & 344 &
\end{tabular}

of research is to develop a general method to compute $s(r)$ or a bound on $s(r)$ for any $r \geq 44$. So far, for any $r(r \geq 44)$, we only know (Greaves et al., 2016, Corollary 2.8)

$$
\frac{32 r^{2}+328 r+296}{1089} \leq s(r) \leq \frac{r(r+1)}{2} .
$$

Here the upper bound $r(r+1) / 2$ is known as the famous Gerzon's bound (from private discussions with Gerzon mentioned in (Lemmens and Seidel, 1973)). It is also well-known that Gerzon's upper bound can be sharpened considerably for certain $r$. For instance, $s(43)=344<(43 \times 44) / 2=946$.

One possible way to get a non-trivial upper bound is to consider $s_{\alpha}(r)$ for a fixed angle $\alpha$. A nice classical result is that $s(r)(r>3)$ can be solved by determining finitely many $s_{\alpha}(r)$ where $1 / \alpha$ is an odd integer bounded by $\sqrt{2 r}$ (Proposition 1). Thus, throughout the paper, we assume $1 / \alpha$ is an odd integer which is greater than 3 . An upper bound for $s_{\alpha}(r)$ is often called a relative bound. (We note that in some papers, relative bound refers specifically to the analog of Gerzon's bound for a particular angle.) Another theorem of note is Theorem 4.5 in (Lemmens and Seidel, 1973), which determines $s_{1 / 3}(r)$ completely by decomposing an equiangular set into pillars (see the precise definition in Section 3.4.1) and studying the algebraic structure of each pillar and also the combinatorial structure when all pillars are non-empty. However, by the same spirit, the next interesting case $\alpha=1 / 5$ is only partially solved and there is a long-standing conjecture (Lemmens and Seidel, 1973, Conjecture 5.8). The best known $s_{1 / 5}(r)$ is summarized in (Greaves et al., 2016, Table 4). For $\alpha \leq 1 / 7$, following the classical method, one might need to characterize the connected simple graphs with maximum eigenvalue 3 (or $>3$ ). The last sentence in (Neumaier, 1989) says this requires substantially stronger techniques. The good news is that relative bounds for general $\alpha$ can be computed by semidefinite programming (SDP) (Barg and Yu, 2014). The best known non-trivial relative bounds and upper bound of $s(r)$ for $44 \leq r \leq 136$ that existed before this paper was originally released can be found in (Barg and Yu, 2014, Table 3). Notice that for $r>136$, this SDP method might give a bound which is greater than Gerzon's bound.

Contributions. In this paper, our main contribution is a universal procedure for computing a 
non-trivial upper bound for general dimension $r$. Our main contributions have three stages, see $(\mathrm{C} 1),(\mathrm{C} 2)$, and (C3).

(C1) We derive a procedure (Subsection 3.5) for computing an upper bound of an equiangular set $X \subset \mathbb{R}^{r}$ with angle $\alpha$. Our method is to decompose $X$ into finitely many equivalence classes with respect to a fixed $K$-base (Definition [5, a maximal negative clique). Here each equivalence class generalizes the concept of a pillar defined in Lemmens and Seidel, 1973, Page 501, Section 4). We prove the number of equivalence classes in $X$ and an upper bound for each equivalence class (Theorems 3 5 and 6).

(C2) After a more careful analysis of the pillar decompositions (Theorems 3 [5, and 6), we provide new relative bounds for the angle $\alpha=1 / 5$ (Theorem 7 and Corollary 4).

(C3) By applying Theorems 3, 5, 6, and 7, we compute upper bounds of equiangular sets for $44 \leq r \leq 400$. The computational results show an explicit upper bound:

$$
\begin{cases}\frac{4 r(m+1)(m+2)}{(2 m+3)^{2}-r}, & r=44,45,46,76,77,78,117,118,166,222,286,358 \\ \frac{\left((2 m+1)^{2}-2\right)\left((2 m+1)^{2}-1\right)}{2}, & \text { other } r \text { between } 44 \text { and } 400 .\end{cases}
$$

This bound is strictly less than Gerzon's bound if $r+2$ is not a square of an odd number (Theorem 8). The result leads us to a conjecture on a new general upper bound (Conjecture1).

We note that our approach using the pillar decomposition can be seen as being similar to the methods of (Balla et al., 2018), which were independently developed. In (Balla et al., 2018), given an equiangular set, a weighted graph with vertices corresponding to vectors in the set and edge weights being the values of the corresponding inner products is constructed. Using Ramsey theory, for large enough graphs, there must exist a clique of positive edges. They proceed using orthogonal projections onto certain sets, like the orthogonal complement of the large clique, to transform their problem about equiangular lines to a related problem about certain two-angle spherical codes. (See also (Gosselin, 2004).) Our methods are related to finding maximal negative cliques and characterizing inner products connecting to the clique via orthogonal projections. The size of positive cliques is bounded by the dimension and negative cliques by a function of the angle, thus yielding a very different structure. The main theorem of their paper concerning equiangular lines is a collection of asymptotic bounds, namely:

Theorem 1. Balla et al., 2018, Theorem 1.1) Fix $\alpha \in(0,1)$. For $r$ sufficiently large relative to $\alpha$, the maximum number of lines in $\mathbb{R}^{r}$ with angle $\alpha$ is exactly $2 r-2$ if $\alpha=1 / 3$ and at most $1.93 r$ otherwise.

At first glance, this seems to contradict (2),$\left(32 r^{2}+328 r+296\right) / 1089 \leq s(r) \leq r(r+1) / 2$ for $r \geq 44$; however, it is important to note that this bound in Theorem 1 is asymptotic in dimension relative to a fixed $\alpha$. That is, for large enough $r$ (where "large enough" depends on $\alpha$ ) $s_{\alpha}(r) \leq 2 r-2$; however, it is not true in general that $s(r) \leq 2 r-2$ holds for large enough $r$. As an example, we can see from our computational results (King and Tang, 2018, Table.pdf) or in Figure 1 that the upper bound on the size of an equiangular set of vectors in $\mathbb{R}^{r}$ for $44 \leq r \leq 400$ with angle $1 / 5$ grows relatively slowly with $r$, while at the same time being significantly smaller than the known bounds for smaller angles. 
Organization. The rest of the paper is organized as follows. In Section 2 , we review the definition of spherical two-distance sets and the fact that an upper bound of a spherical two-distance set can be solved by semidefinite programming (SDP) (Barg and Yu, 2013). In Section 3 we decompose a given equiangular set $X$ into finitely many equivalence classes with respect to a fixed $K$-base (Definitions 556). According to the size of a $K$-base, we show the number of equivalence classes in $X$ and an upper bound for each equivalence class (Theorems 3 , 5 and 6). We also provide a procedure (Subsection 3.5) and illustrate an example (Example 1) for computing an upper bound for given dimension $r$ and angle $\alpha$. By further analyzing the pillar decomposition in Section 4 , we are able to prove a new relative bound for the angle $\alpha=1 / 5$ (Theorem 7 and Corollary 4). In Section 5 , by applying the procedure, we compute upper bounds for the dimensions $44 \leq r \leq 400$ and conclude the computational results in Theorem 8 . In Section 6 , we compare the new (computable) relative bounds for $\alpha=1 / 5,1 / 7$ and the SDP bounds (Barg and Yu, 2013, Theorem 3.1) by experiments. We also interpret the experimental/computational details in this section. The results in Sections 3 and 4 which require technical proofs are proven in the order they appear in the text in Appendices A D.

\section{Review of spherical two-distance sets and SDP bounds}

In this section, we review spherical two-distance sets and the fact that an upper bound of a spherical two-distance set can be determined by semidefinite programming (SDP). A spherical twodistance set is a more general concept than an equiangular set. The SDP method is closely related to Delsarte's method (Delsarte et al., 1977; Musin, 2009) and harmonic analysis in coding theory (Bachoc and Vallentin, 2008). We provide (Yu, 2014) as a good survey for the interested readers since we will only repeat the key results without proof here. The main point we hope to highlight here is that an upper bound of equiangular sets in $\mathbb{R}^{r}$ is computable for any $r>3$, see Theorem 2 and Proposition 1 below.

Definition 2. We say $X=\left\{x_{1}, \ldots, x_{s}\right\} \subset \mathbb{R}^{r}$ is a spherical two-distance set with mutual inner products $\alpha, \beta$ if

$$
\left\langle x_{i}, x_{j}\right\rangle= \begin{cases}1, & i=j \\ \alpha \text { or } \beta, & i \neq j .\end{cases}
$$

For any spherical two-distance set $X$, we use the notation $|X|$ to denote the cardinality of $X$. We use the notation $s(r, \alpha, \beta)$ to denote the maximum cardinality of a spherical two-distance set with mutual inner products $\alpha, \beta$ in $\mathbb{R}^{r}$.

Theorem 2. Barq and Yu, 2013, Theorem 3.1) Suppose $X \subset \mathbb{R}^{r}$ is a spherical two-distance set with mutual inner products $\alpha, \beta$. An upper bound of $|X|$ is given by the solution of a semidefinite programming (SDP) problem.

The concrete SDP formulation can be found in (Barg and Yu, 2013, Theorem 3.1). As we can see, an equiangular set $X$ in $\mathbb{R}^{r}$ with angle $\alpha$ is a special spherical two-distance set with mutual inner products $\alpha,-\alpha$; that is, $s_{\alpha}(r)=s(r, \alpha,-\alpha)$. So an upper bound of $|X|$ for a given $\alpha$ can be computed by running SDP tools. Proposition 1, which is a direct corollary of (Larman et al., 1977, Theorem 2), shows an upper bound of $|X|$ is either $2 r+3$ or given by $s_{\alpha}(r)$ with $\alpha=1 /(2 L-1)$ for finitely many possible $L$ 's. 
Proposition 1. For any equiangular set $X \subset \mathbb{R}^{r}$ with angle $\alpha$, if $|X|>2 r+3$, then $\alpha=1 /(2 L-1)$ for some $L$ such that $2 \leq L \leq(1+\sqrt{2 r}) / 2$.

We remark that $|X|<2 r-2(<2 r+3)$ when $\alpha=1 /(2 \cdot 2-1)=1 / 3$ and $r \geq 15$ (Lemmens and Seidel, 1973, Theorem 4.5). Thus by Theorem 2 and Proposition 1, for any $r \geq 15$, an upper bound of $|X|$ is either $2 r+3$ or the maximum of the SDP bounds for $\alpha=1 / 5, \ldots, 1 /(2 L-1)$. Big progress was made in this spirit in proving the relative bound 276 for $24 \leq r \leq 60$ and $\alpha=1 / 5$ (Barg and Yu, 2014). However, it is seen that the SDP bounds for $\alpha=1 / 5$ are greater than Gerzon's bound for $r=137-139$ (Barg and Yu, 2014, Table 3). We also provide evidence in Section 6 that for $\alpha=1 / 5,1 / 7$, the SDP bound does not guarantee a non-trivial upper bound if $r$ is sufficiently large, and we expect similar behavior for smaller values of $\alpha$. In the rest of the paper, we focus on improving the relative bounds with help of the pillar decomposition. We remark that all our results except Theorem 3 hold for any $0<\alpha<1$. However, we are only interested in the $\alpha$ 's such that $1 / \alpha$ is an odd number greater or equal to 3 .

\section{Pillar decomposition}

\subsection{Gramian matrices}

For a set of vectors $X=\left\{x_{1}, \ldots, x_{s}\right\}$ in $\mathbb{R}^{r}(r \geq 2)$, we also denote by $X$ the $r \times s$ matrix with $x_{1}, \ldots, x_{s}$ as its column vectors. We begin by defining the Gramian matrix of $X$.

Definition 3. For any $X=\left\{x_{1}, \ldots, x_{s}\right\} \subset \mathbb{R}^{r}$, the Gramian matrix of $X$, denoted by $G\left(x_{1}, \ldots, x_{s}\right)$ or $G(X)$, is the matrix of mutual inner products of $x_{1}, \ldots, x_{s}$, namely

$$
G(X)=X^{\top} X=\left(\begin{array}{cccc}
\left\langle x_{1}, x_{1}\right\rangle & \left\langle x_{1}, x_{2}\right\rangle & \ldots & \left\langle x_{1}, x_{s}\right\rangle \\
\left\langle x_{2}, x_{1}\right\rangle & \left\langle x_{2}, x_{2}\right\rangle & \ldots & \left\langle x_{2}, x_{s}\right\rangle \\
\vdots & \vdots & \ddots & \vdots \\
\left\langle x_{s}, x_{1}\right\rangle & \left\langle x_{s}, x_{2}\right\rangle & \ldots & \left\langle x_{s}, x_{s}\right\rangle
\end{array}\right)_{s \times s}
$$

It follows directly from Definition 3 that $G(X)$ is symmetric and positive semidefinite for any finite set $X \subset \mathbb{R}^{r}$. The following lemma is standard and shows that $X$ is a set of linearly independent vectors if and only if $G(X)$ is non-singular.

Lemma 1 (see, for example, (Horn and Johnson, 2012)). For any $X=\left\{x_{1}, \ldots, x_{s}\right\} \subset \mathbb{R}^{r}$, $\operatorname{rank} G(X)=\operatorname{dim} \operatorname{span} X$. Further, a linear dependence relation of the columns is one of the vectors and vice versa.

\subsection{Switching equivalent equiangular sets}

Recall from the introduction that we denote equiangular lines in $\mathbb{R}^{r}$ by a finite set of unit equiangular vectors $X=\left\{x_{1}, \ldots, x_{s}\right\}$. We note that when passing from lines to vectors, we must make a choice of one of the two unit vectors which span the same line. In particular, two unit vectors $-x_{i}$ and $x_{i}$ denote the same line. However, this choice affects the signs of the inner products. If two sets of vectors represent the same set of lines, we say they are switching equivalent (Van Lint and Seidel, 1966; Godsil and Royle, 2001). More generally and more precisely, we have the definition below. 
Definition 4. Two sets of unit vectors $X$ and $Y$ in $\mathbb{R}^{r}$ are switching equivalent if there exist a diagonal $(1,-1)$-matrix $B$ and a permutation matrix $C$ such that

$$
(C B)^{\top} \cdot G(X) \cdot(C B)=G(Y)
$$

We also say $G(X)$ is switching equivalent to $G(Y)$, denoted by $G(X) \cong G(Y)$.

Lemma 2. Let $X$ and $Y$ be two sets of unit vectors in $\mathbb{R}^{r}$. If $G(X) \cong G(Y)$, then $G(X)$ and $G(Y)$ have the same eigenvalues.

Proof. If $G(X) \cong G(Y)$, then by Definition 4, they are orthogonally similar and hence have the same eigenvalues.

Lemma 3. Let $X$ and $Y$ in $\mathbb{R}^{r}$ be two equiangular sets with the same angle $\alpha$. If $|X|=|Y|$ and we can order the vectors in $X$ and $Y$ as $\left\{x_{1}, \ldots, x_{s}\right\}$ and $\left\{y_{1}, \ldots, y_{s}\right\}$ respectively such that $x_{i}= \pm y_{i}$ for $i=1, \ldots, s$, then $X$ and $Y$ are switching equivalent (i.e., $G(X) \cong G(Y))$.

Proof. Note there exist a diagonal $(1,-1)$-matrix $B$ and a permutation matrix $C$ such that $X C B=$ $Y$ and hence $(C B)^{\top} \cdot G(X) \cdot(C B)=G(Y)$. By Definition $4 X$ and $Y$ are switching equivalent.

We further remark that if $G(X) \cong G(Y)$, there need not exist a diagonal $(1,-1)$-matrix $B$ and a permutation matrix $C$ such that $X C B=Y$. For example, if $O$ is an orthogonal matrix, then $G(O X)=G(X)$. The key idea is that given a positive semidefinite matrix $G$, we can always factor it (via, for example, an eigendecomposition) as $G=X^{T} X$ so that $G=G(X)$. Thus we will work on the level of Gramian matrices.

\subsection{Base size and $K$-base}

Before we investigate more structures associated to equiangular sets, we provide a basic fact (Lemma 4) from linear algebra. Following Seidel's spirit, we will often decompose matrices using building blocks of the $s \times s$ identity matrix $I_{s}$ and the $s \times s$ all-one matrix $J_{s}$, which we will denote by $I$ and $J$, respectively, when $s$ is clear from context.

Lemma 4. Consider an $s \times s$ matrix $G$. If all diagonal entries of $G$ are the same, say a, and all off-diagonal entries are the same, say $b$, then $G=(a-b) I_{s}+b J_{s}$, and hence $G$ has a simple eigenvalue $\lambda_{1}=a+(s-1) b$ and an eigenvalue $\lambda_{2}=a-b$ with multiplicity $s-1$.

Following the above lemma, we present an easy observation about the structure of Gramian matrices. As what has been pointed out at the very beginning of (Lemmens and Seidel, 1973, Section 4), we have Proposition 2 . Here we generalize the original setting in (Lemmens and Seidel, 1973) since we consider the Gramian matrices of switching equivalent equiangular sets.

Proposition 2. If there exist $k \geq 2$ equiangular vectors $p_{1}, \ldots, p_{k}$ with angle $\alpha$ such that

$$
G\left(p_{1}, \ldots, p_{k}\right) \cong(1+\alpha) I-\alpha J
$$

then $k \leq(1 / \alpha)+1$. Furthermore, if $k<(1 / \alpha)+1$, then the vectors $p_{1}, \ldots, p_{k}$ are linearly independent, and if $k=(1 / \alpha)+1$, then the vectors $p_{1}, \ldots, p_{k}$ are linearly dependent. When $k=(1 / \alpha)+1$ and $G\left(p_{1}, \ldots, p_{k}\right)=(1+\alpha) I-\alpha J$, the linear dependence relation is $\sum_{j=1}^{k} p_{j}=0$. 
Proof. By Lemma $2 G\left(p_{1}, \ldots, p_{k}\right)$ and $(1+\alpha) I-\alpha J$ have same eigenvalues and thus by Lemma 4. $G\left(p_{1}, \ldots, p_{k}\right)$ has a simple eigenvalue $\lambda_{1}=1-(k-1) \alpha$ and an eigenvalue $\lambda_{2}=1+\alpha>0$ with multiplicity $k-1$. Since the Gramian matrix is positive semidefinite, the eigenvalue $\lambda_{1}$ should be non-negative. So

$$
\lambda_{1}=1-(k-1) \alpha \geq 0 \text { or, equivalently, } k \leq(1 / \alpha)+1 .
$$

Furthermore, if $k<(1 / \alpha)+1$, then the eigenvalue $\lambda_{1}$ satisfies

$$
\lambda_{1}=1-(k-1) \alpha=\alpha((1 / \alpha)+1-k)>0 .
$$

Both eigenvalues are nonzero, so the Gramian matrix is full rank and hence by Lemma $1, p_{1}, \ldots, p_{k}$ are linearly independent. If $k=(1 / \alpha)+1$, we similarly have $\lambda_{1}=0$. So $p_{1}, \ldots, p_{k}$ are linearly dependent. If further $G\left(p_{1}, \ldots, p_{k}\right)=(1+\alpha) I-\alpha J$, then the all-ones vectors is an eigenvector for the eigenvalue 0, yielding the linear dependence relation (Lemma 1).

Proposition 2 inspires two new concepts: base size and $K$-base (Definition 5 ).

Definition 5. Let $X \subset \mathbb{R}^{r}$ be an equiangular set with angle $\alpha$. The base size of $X, K_{\alpha}(X)$ is defined as

$$
K_{\alpha}(X) \triangleq \max \left\{k \in \mathbb{N}^{+} \mid \exists p_{1}, \ldots, p_{k} \in X \text { s.t. } G\left(p_{1}, \ldots, p_{k}\right) \cong(1+\alpha) I-\alpha J\right\} .
$$

Let $K=K_{\alpha}(X)$. If $K$ vectors $p_{1}, \ldots, p_{K} \in X$ satisfy $G\left(p_{1}, \ldots, p_{K}\right) \cong(1+\alpha) I-\alpha J$, then we say $\left\{p_{1}, \ldots, p_{K}\right\}$ is a $K$-base of $X$.

It follows from Definitions 4 and 5 that if $\left\{p_{1}, \ldots, p_{K}\right\}$ is a $K$-base of $X$, then $\left\{p_{1}, \ldots, p_{K}\right\}$ is switching equivalent to an equiangular set $\left\{\tilde{p}_{1}, \ldots, \tilde{p}_{K}\right\}$, which has $G\left(\tilde{p}_{1}, \ldots, \tilde{p}_{K}\right)=(1+\alpha) I-\alpha J$, and further $X$ is then also switching equivalent to $\left(X \backslash\left\{p_{1}, \ldots, p_{K}\right\}\right) \bigcup\left\{\tilde{p}_{1}, \ldots, \tilde{p}_{K}\right\}$. Thus we assume without loss of generality that

$$
G\left(p_{1}, \ldots, p_{K}\right)=(1+\alpha) I-\alpha J .
$$

That means for any $1 \leq i<j \leq K,\left\langle p_{i}, p_{j}\right\rangle=-\alpha$. Using graph theory terminology, a $K$-base is a negative clique. Lemma 2 shows that the size of a $K$-base, that is $K_{\alpha}(X)$, is at most $(1 / \alpha)+1$. We next show that $K_{\alpha}(X)$ is at least 2 if $|X| \geq 2$ (Proposition 3).

Proposition 3. Let $X \subset \mathbb{R}^{r}$ be an equiangular set with angle $\alpha$. If $|X| \geq 2$, then $K_{\alpha}(X) \geq 2$.

Proof. Take two different vectors $x_{1}, x_{2} \in X$. If $\left\langle x_{1}, x_{2}\right\rangle=-\alpha$, then by Definition $5 K_{\alpha}(X) \geq 2$. If $\left\langle x_{1}, x_{2}\right\rangle=\alpha$, then $\left\langle-x_{1}, x_{2}\right\rangle=-\alpha$. By Lemma 3 , $\left\{x_{1}, x_{2}\right\}$ is switching equivalent to $\left\{-x_{1}, x_{2}\right\}$. So $G\left(x_{1}, x_{2}\right) \cong G\left(-x_{1}, x_{2}\right)=(1+\alpha) I-\alpha J$. Again, by Definition $K_{\alpha}(X) \geq 2$.

\subsection{Equivalence classes w.r.t. a K-base}

Let $X$ in $\mathbb{R}^{r}$ be an equiangular set with angle $\alpha$. Let $K=K_{\alpha}(X)$. We fix a $K$-base $\left\{p_{1}, \ldots, p_{K}\right\}$ of $X$ satisfying the condition (44). Let $P$ be the linear subspace of $\mathbb{R}^{r}$ spanned by $p_{1}, \ldots, p_{K}$ and let $P^{\perp}$ be the orthogonal complement of $P$ in $\mathbb{R}^{r}$. For any $x \in X \backslash\left\{p_{1}, \ldots, p_{K}\right\}$, we first present Proposition 4 to describe the projection of $x$ onto the subspace $P$. Note that part (1) originally comes from (Lemmens and Seidel, 1973). 
Proposition 4. For any $x \in X \backslash\left\{p_{1}, \ldots, p_{K}\right\}$, suppose $\left(\left\langle x, p_{1}\right\rangle, \ldots,\left\langle x, p_{K}\right\rangle\right)^{\top}=\alpha \cdot \epsilon$, where $\epsilon$ is $a(1,-1)$-vector in $\mathbb{R}^{K}$, namely

$$
\epsilon=\left(\epsilon^{(1)}, \ldots, \epsilon^{(K)}\right)^{\top}, \epsilon^{(i)}= \pm 1 .
$$

If we decompose $x$ in $P$ and $P^{\perp}$ as $x=h+c$, where $h \in P$ and $c \in P^{\perp}$, then

(1) for $K=(1 / \alpha)+1, h=\frac{1}{K}\left(\epsilon^{(1)} p_{1}+\cdots+\epsilon^{(K)} p_{K}\right)$ and $\langle h, h\rangle=\alpha$, and

(2) for $K<(1 / \alpha)+1, h=a^{(1)} p_{1}+\cdots+a^{(K)} p_{K}$, where

$$
\left(a^{(1)}, \ldots, a^{(K)}\right)^{\top}=\alpha((1+\alpha) I-\alpha J)^{-1} \epsilon,
$$

and $\langle h, h\rangle$ is given by

$$
\ell(K, n)=\alpha^{2} \frac{4 \alpha \cdot n \cdot(n-K)+(1+\alpha) K}{(1+\alpha)(1+\alpha-K \alpha)},
$$

where $n$ is the number of positive signs among $\epsilon^{(1)}, \ldots, \epsilon^{(K)}$. Furthermore, we have

$$
\begin{cases}0<\ell(K, n)<\alpha, & K-\frac{(1 / \alpha)+1}{2}<n \leq\lfloor K / 2\rfloor \\ \ell(K, n)=\alpha, & n=K-\frac{(1 / \alpha)+1}{2} \\ \alpha<\ell(K, n)<1, & 1 \leq n<K-\frac{(1 / \alpha)+1}{2}\end{cases}
$$

Proof. Part (1) was shown in (Lemmens and Seidel, 1973, pages 501-502). We only prove part (2). By Proposition 2 if $K<(1 / \alpha)+1$, then $p_{1}, \ldots, p_{K}$ are linearly independent. So $p_{1}, \ldots, p_{K}$ form a basis of $P$. Since $h \in P$, we can assume $h=\sum_{j=1}^{K} a^{(j)} p_{j}$. Note by (4), $\left\langle p_{i}, p_{j}\right\rangle=-\alpha(i \neq j)$. So for each $i=1, \ldots, K$, we have

$$
\left\langle h, p_{i}\right\rangle=\left\langle\sum_{j=1}^{K} a^{(j)} p_{j}, p_{i}\right\rangle=a^{(i)} \cdot\left\langle p_{i}, p_{i}\right\rangle+\sum_{j \neq i} a^{(j)} \cdot\left\langle p_{j}, p_{i}\right\rangle=a^{(i)}-\alpha \cdot \sum_{j \neq i} a^{(j)} .
$$

On the other hand, since $x=h+c, h \in P$ and $c \in P^{\perp}$, we have

$$
\left\langle h, p_{i}\right\rangle=\left\langle x, p_{i}\right\rangle=\alpha \cdot \epsilon^{(i)} .
$$

Note the left-hand sides of equalities (8) and (9) are the same. So we have a system of $K$ linear equations in $a^{(1)}, \cdots, a^{(K)}$ which has a unique solution

$$
a=\left(a^{(1)}, \ldots, a^{(K)}\right)^{\top}=\alpha((1+\alpha) I-\alpha J)^{-1} \epsilon .
$$

We denote the $r \times K$ matrix with column vectors $p_{1}, \ldots, p_{K}$ by $\left(\begin{array}{cccc}p_{1} & p_{2} & \ldots & p_{K}\end{array}\right)$. Then it is 
straightforward to calculate by linear algebra that

$$
\begin{aligned}
\langle h, h\rangle= & \left\langle\sum_{j=1}^{K} a^{(j)} p_{j}, \sum_{j=1}^{K} a^{(j)} p_{j}\right\rangle \\
= & a^{\top}\left(\begin{array}{llll}
p_{1} & p_{2} & \ldots & p_{K}
\end{array}\right)^{\top}\left(\begin{array}{llll}
p_{1} & p_{2} & \ldots & p_{K}
\end{array}\right) a \\
= & a^{\top}((1+\alpha) I-\alpha J) a \\
= & \alpha \epsilon^{\top}\left(((1+\alpha) I-\alpha J)^{-1}\right)^{\top}((1+\alpha) I-\alpha J) \alpha((1+\alpha) I-\alpha J)^{-1} \epsilon \\
= & \alpha^{2} \epsilon^{\top}((1+\alpha) I-\alpha J)^{-1} \epsilon \\
= & \alpha^{2} \epsilon^{\top} \frac{(1+\alpha-K \alpha) I+\alpha J}{(1+\alpha)(1+\alpha-K \alpha)} \epsilon \\
= & \frac{\alpha^{2}}{(1+\alpha)(1+\alpha-K \alpha)}\left((1+\alpha-K \alpha) \epsilon^{\top} \epsilon+\alpha \epsilon^{\top} J \epsilon\right) \\
& =\frac{\alpha^{2}}{(1+\alpha)(1+\alpha-K \alpha)}\left((1+\alpha-K \alpha) K+\alpha(2 n-K)^{2}\right) \\
& =\alpha^{2} \frac{4 \alpha \cdot n \cdot(n-K)+(1+\alpha) K}{(1+\alpha)(1+\alpha-K \alpha)},
\end{aligned}
$$

where the second to last equality follows from the two facts

$$
\epsilon^{\top} \epsilon=\sum_{i=1}^{K}\left(\epsilon^{(i)}\right)^{2}=K \quad \text { and } \quad \epsilon^{\top} J \epsilon=\left(\sum_{i=1}^{K} \epsilon^{(i)}\right)^{2}=(n-(K-n))^{2}=(2 n-K)^{2} .
$$

Furthermore, in an equiangular set with angle $\alpha$, no two vectors can be orthogonal since $\alpha \neq 0$ (note $0<\alpha<1$ by Definition (1). Thus no vector in $X$ can lie in the orthogonal complement $P^{\perp}$ of the $K$-base and $\ell(K, n)=\langle h, h\rangle>0$. By (10),

$$
\ell(K, n)=\alpha^{2} \frac{4 \alpha(n-K / 2)^{2}+K(1+\alpha-K \alpha)}{(1+\alpha)(1+\alpha-K \alpha)} .
$$

Thus, as a function of $n, \ell(K, n)$ is strictly increasing over $1 \leq n \leq K / 2$ with

$$
\ell\left(K, K-\frac{(1 / \alpha)+1}{2}\right)=\alpha \text { and } \ell(K,\lfloor K / 2\rfloor) \leq \ell(K, K / 2)=\alpha^{2} \frac{K}{1+\alpha}<1 .
$$

So we have proved (7).

Definition 6. For any $x, y \in X \backslash\left\{p_{1}, \ldots, p_{K}\right\}$, suppose

$$
x=h_{1}+c_{1} \text { and } y=h_{2}+c_{2} \text {, where } h_{1}, h_{2} \in P \text { and } c_{1}, c_{2} \in P^{\perp} .
$$

We say $x \sim y$ w.r.t. $\left\{p_{1}, \ldots, p_{K}\right\}$ if $h_{1}$ and $h_{2}$ are linearly dependent. The resulting equivalence classes w.r.t. $\left\{p_{1}, \ldots, p_{K}\right\}$ are

$$
\bar{x}=\left\{y \in X \backslash\left\{p_{1}, \ldots, p_{K}\right\} \mid x \sim y\right\}, \quad \forall x \in X \backslash\left\{p_{1}, \ldots, p_{K}\right\} .
$$


Remark 1. Each equivalence class $\bar{x}$ is exactly a "pillar" of h defined in (Lemmens and Seide, 1973, Page 501, Section 4). However, with the exception of the fairly trivial (Lemmens and Seide), 1973, Theorem 4.4), only the extremal case $K_{\alpha}(X)=(1 / \alpha)+1$ is discussed. Our discussion here is more general for any possible $K_{\alpha}(X)$.

Lemma 5. For any $x, y \in X \backslash\left\{p_{1}, \ldots, p_{K}\right\}$, suppose $x=h_{1}+c_{1}$ and $y=h_{2}+c_{2}$ with

$$
\left(\left\langle x, p_{1}\right\rangle, \ldots,\left\langle x, p_{K}\right\rangle\right)^{\top}=\alpha \cdot \epsilon_{1}, \quad \text { and }\left(\left\langle y, p_{1}\right\rangle, \ldots,\left\langle y, p_{K}\right\rangle\right)^{\top}=\alpha \cdot \epsilon_{2},
$$

where $h_{1}, h_{2} \in P, c_{1}, c_{2} \in P^{\perp}$ and $\epsilon_{1}, \epsilon_{2}$ are $(1,-1)$-vectors in $\mathbb{R}^{K}$. Then the three statements below are equivalent
(i) $x \sim y$;
(ii) $\epsilon_{2}= \pm \epsilon_{1}$;
(iii) $h_{1}= \pm h_{2}$.

Hence for each equivalence class $\bar{x}$, there exists an $\epsilon_{1}$ such that

$$
\bar{x}=\left\{x \in X \backslash\left\{p_{1}, \ldots, p_{K}\right\} \mid\left(\left\langle x, p_{1}\right\rangle, \ldots,\left\langle x, p_{K}\right\rangle\right)^{\top}= \pm \alpha \cdot \epsilon_{1}\right\} .
$$

Proof. Assume that $K<(1 / \alpha)+1$. It follows from (5) in Proposition 4 that for $j=1,2$

$$
h_{j}=\left(\begin{array}{llll}
p_{1} & p_{2} & \ldots & p_{K}
\end{array}\right) \alpha((1+\alpha) I-\alpha J)^{-1} \epsilon_{j},
$$

where the matrix on the left-hand-side of the product $\left(\begin{array}{llll}p_{1} & p_{2} & \ldots & p_{K}\end{array}\right)$ is the $r \times K$ matrix with column vectors $p_{1}, \ldots, p_{K}$. Thus, there exists a real number $\lambda \neq 0$ such that $h_{1}=\lambda h_{2}$ if and only if

$$
0=h_{1}-\lambda h_{2}=\left(\begin{array}{llll}
p_{1} & p_{2} & \ldots & p_{K}
\end{array}\right) \alpha((1+\alpha) I-\alpha J)^{-1}\left(\epsilon_{1}-\lambda \epsilon_{2}\right) .
$$

If $K=(1 / \alpha)+1$, the argument is similar, but additionally makes use of the fact that in this case

$$
\operatorname{kern}\left(\begin{array}{llll}
p_{1} & p_{2} & \ldots & p_{K}
\end{array}\right)=\operatorname{span}\left(\begin{array}{llll}
1 & 1 & \ldots & 1
\end{array}\right)^{\top} .
$$

Corollary 1. Each equivalence classe $\bar{x}$ w.r.t. $\left\{p_{1}, \ldots, p_{K}\right\}$ is switching equivalent to an equiangular set $Y=\left\{y_{1}, \ldots, y_{s}\right\}$ such that there exist $h \in P$ and a $(1,-1)$-vector $\epsilon \in \mathbb{R}^{K}$ such that for every $y_{i}$ $(i=1, \ldots, s)$, we have

$$
y_{i}=h+d_{i}, \quad \text { where } d_{i} \in P^{\perp} \quad \text { and } \quad\left(\left\langle y_{i}, p_{1}\right\rangle, \ldots,\left\langle y_{i}, p_{K}\right\rangle\right)^{\top}=\alpha \cdot \epsilon .
$$

Proof. Let $\bar{x}=\left\{x_{1}, \ldots, x_{s}\right\}$. For each $i=1, \ldots, s, x_{i}$ can be writen as

$$
x_{i}=h_{i}+c_{i}, \quad \text { where } h_{i} \in P \text { and } c_{i} \in P^{\perp} .
$$

By Lemma 5 , there exists $h \in P$ such that $h_{i}=h$, or $-h$ for each $i=1, \ldots, s$. Let

$$
Y=\left\{x_{i} \mid x_{i}=h+c_{i}, x_{i} \in \bar{x}\right\} \cup\left\{-x_{i} \mid x_{i}=-h+c_{i}, x_{i} \in \bar{x}\right\} .
$$

Note that $Y$ is switching equivalent to $\bar{x}$. Rename the vectors in $Y$ as $y_{1}, \ldots, y_{s}$. Then we can write each of them as

$$
y_{i}=h+d_{i}, \quad \text { where } h \in P \text { and } d_{i} \in P^{\perp} .
$$

So for any $i,\left(\left\langle y_{i}, p_{1}\right\rangle, \ldots,\left\langle y_{i}, p_{K}\right\rangle\right)^{\top}=\left(\left\langle h, p_{1}\right\rangle, \ldots,\left\langle h, p_{K}\right\rangle\right)^{\top}=\alpha \cdot \epsilon$ for a $(1,-1)$-vector $\epsilon$. 
By the definition of equivalence classes w.r.t. $\left\{p_{1}, \ldots, p_{K}\right\}$ (Definition 6 ), we have $X=\bigcup_{x \in X} \bar{x}$. In order to derive an upper bound of $|X|$, we naturally have two questions below.

(Question 1). How many (necessarily finitely many) equivalence classes are there in $X$ ?

(Question 2). For each equivalence class $\bar{x} \subset X$, what is an upper bound for $|\bar{x}|$ ?

We answer the two questions in the following subsections according to two different cases $K_{\alpha}(X)=$ $(1 / \alpha)+1\left(\right.$ Theorem 3) and $K_{\alpha}(X)<(1 / \alpha)+1$ (Theorems 5 and $[$ ).

\subsection{1. $K_{\alpha}(X)=(1 / \alpha)+1$}

The extremal case $K_{\alpha}(X)=(1 / \alpha)+1$ was studied in detail in (Lemmens and Seidel, 1973). In this case, $\left\{p_{1}, p_{2}, \ldots, p_{K}\right\}$ forms an equiangular tight frame for its span and can be thought of as vectors pointing to vertices of a regular simplex centered at the origin (Fickus et al., 2017). (Recall $\sum_{j=1}^{K} p_{j}=0$ by Proposition 2) For this case, we answer (Question 1) and (Question 2) in Theorem 3. The proof is given in Appendix A

Theorem 3. Let $X \subset \mathbb{R}^{r}$ be an equiangular set with angle $\alpha$, where $1 / \alpha$ is an odd number greater or equal to 3 . If $K=K_{\alpha}(X)=(1 / \alpha)+1$, then there are $\frac{1}{2}\left(\begin{array}{c}K \\ K / 2\end{array}\right)$ equivalence classes $\bar{x}$ w.r.t. any fixed $K$-base, and for each $\bar{x}$,

$$
|\bar{x}| \leq r-K+1+\left\lfloor 2 \alpha \frac{r-K+1}{1-\alpha}\right\rfloor .
$$

Hence,

$$
|X| \leq K+\frac{1}{2}\left(\begin{array}{c}
K \\
K / 2
\end{array}\right)\left(r-K+1+\left\lfloor 2 \alpha \frac{r-K+1}{1-\alpha}\right\rfloor\right) .
$$

For $\alpha=1 / 3$ and $1 / 5$, the upper bound in Theorem 3 can be reduced significantly by applying spectral graph theory. For instance, if $\alpha=1 / 3,|X| \leq 2(r-1)$ for $r \geq 15$, and any set $X$ which attains this upper bound must have $K_{\alpha}(X)=(1 / \alpha)+1=4$ (Lemmens and Seidel, 1973, Theorem 4.5). What is deeply hidden in its proof is that the only connected simple graph with maximum eigenvalue 1 is the complete graph on two vertices. Theorem 4 below is proved by the fact that the connected simple graphs with maximum eigenvalue 2 only have 5 patterns (Lemmens and Seidel, 1973, Theorem 5.1).

Theorem 4. (Lemmens and Seided, 1979, Theorem 5.7) Let $X \subset \mathbb{R}^{r}$ be an equiangular set with angle $1 / 5$. If $K_{1 / 5}(X)=6$, then

$$
|X| \leq \begin{cases}276, & 23 \leq r \leq 185 \\ r+1+\lfloor(r-5) / 2\rfloor, & r \geq 185 .\end{cases}
$$

3.4.2. $K_{\alpha}(X)<(1 / \alpha)+1$

Let $X \subset \mathbb{R}^{r}$ be an equiangular set with angle $\alpha$. In this subsection, we answer (Question 1) and (Question 2) for the case $K=K_{\alpha}(X)<(1 / \alpha)+1$. When $\alpha$ and a $K$-base $\left\{p_{1}, \ldots, p_{K}\right\}$ are fixed, we notice that for any vector $x \in X \backslash\left\{p_{1}, \ldots, p_{K}\right\}$, the norm of its projection $h$ onto the subspace $P$ (spanned by $p_{1}, \ldots, p_{K}$ ), that is the value of $\ell(K, n)$ in (6), only depends on $n$, namely the number of positive inner products among $\left\langle x, p_{1}\right\rangle, \ldots,\left\langle x, p_{K}\right\rangle$. We further note that the function $\ell(K, n)$ is symmetric w.r.t. $n$ and $K-n$. That means when we have $n$ or $K-n$ positive 
signs among $\left\langle x, p_{1}\right\rangle, \ldots,\left\langle x, p_{K}\right\rangle$, the norm of $h$ will be the same. This should be expected, since if $X$ is equiangular, $(X \backslash\{x\}) \cup\{-x\}$ is also equiangular, and these two equiangular sets are switching equivalent. Inspired by these observations, we define subsets $X(K, n)$ of $X \backslash\left\{p_{1}, \ldots, p_{K}\right\}$ for $n=0, \ldots,\lfloor K / 2\rfloor$,

$X(K, n) \triangleq\left\{x \in X \backslash\left\{p_{1}, \ldots, p_{K}\right\} \mid \exists\right.$ exactly $n$ or $K-n$ positive signs among $\left.\left\langle x, p_{1}\right\rangle, \ldots,\left\langle x, p_{K}\right\rangle\right\}$.

Then by the formula (6), for any two distinct vectors $x, y \in X(K, n)$, if we project them onto the subspace $P$, the norms of their projections are the same. We next show by Proposition 5 that $X(K, 0)=\emptyset$.

Proposition 5. Let $X \subset \mathbb{R}^{r}$ be an equiangular set with angle $\alpha$. If $K=K_{\alpha}(X)<(1 / \alpha)+1$, then $X(K, 0)=\emptyset$.

Proof. Assume that $X(K, 0) \neq \emptyset$. For any $x \in X(K, 0)$, by the definition of $X(K, 0)$ in (11), $\left\langle x, p_{1}\right\rangle, \ldots,\left\langle x, p_{K}\right\rangle$ are either all $-\alpha$ or all $\alpha$. That means we have

$$
G\left(x, p_{1}, \ldots, p_{K}\right)=(1+\alpha) I-\alpha J \text { or } G\left(-x, p_{1}, \ldots, p_{K}\right)=(1+\alpha) I-\alpha J .
$$

By Lemma 3 ] $G\left(x, p_{1}, \ldots, p_{K}\right) \cong(1+\alpha) I-\alpha J$. So by the definition of $K_{\alpha}(X)$ in (3),$K+1 \leq$ $K_{\alpha}(X)=K$. This is a contradiction.

Since $X(K, 0)=\emptyset$, we write $X$ as a disjoint union

$$
X=\left\{p_{1}, \ldots, p_{K}\right\} \bigcup\left(X \backslash\left\{p_{1}, \ldots, p_{K}\right\}\right)=\left\{p_{1}, \ldots, p_{K}\right\} \bigcup \bigcup_{n=1}^{\lfloor K / 2\rfloor} X(K, n) .
$$

Below, in Theorem 5 we give the number of equivalence classes $\bar{x}$ in $X(K, n)$ for each $n$, and in Theorem 6, for each equivalence class $\bar{x}$ in $X(K, n)$, we give an upper bound on $|\bar{x}|$ in terms of $r, \alpha, K, \ell(K, n)$. The proof of Theorem 6 is given in Appendix B

Theorem 5. Let $X \subset \mathbb{R}^{r}$ be an equiangular set with angle $\alpha$. Suppose $K=K_{\alpha}(X)<(1 / \alpha)+1$. Fix a $K$-base $\left\{p_{1}, \ldots, p_{K}\right\}$ and define subsets $X(K, n)$ for $n=1, \ldots,\lfloor K / 2\rfloor$ as in (11). For each $n$, if $2 n<K$, then the number of equivalence classes $\bar{x}$ in $X(K, n)$ w.r.t $\left\{p_{1}, \ldots, p_{K}\right\}$ is $\left(\begin{array}{l}K \\ n\end{array}\right)$, and if $2 n=K$, then the number of equivalence classes $\bar{x}$ is $\frac{1}{2}\left(\begin{array}{l}K \\ n\end{array}\right)$.

Proof. $X(K, n)$ is defined in (11) by the set of $x \in X \backslash\left\{p_{1}, \ldots, p_{K}\right\}$ with $n$ or $K-n$ positive inner products among $\left\langle x, p_{1}\right\rangle, \ldots\left\langle x, p_{K}\right\rangle$, whereas it follows from Lemma 5 that the equivalence classes $\bar{x}$ are determined by which specific inner products are positive. Thus the theorem follows from a simple combinatorial argument. The only slight trick is that when $2 n=K$, one notes that when $x \in \bar{x} \in X(2 n, n),-x \in \bar{x}$ as well.

Theorem 6. Let $X \subset \mathbb{R}^{r}$ be an equiangular set with angle $\alpha$. Suppose $K=K_{\alpha}(X)<(1 / \alpha)+1$. Fix a $K$-base $\left\{p_{1}, \ldots, p_{K}\right\}$ and define subsets $X(K, n)$ for $n=1, \ldots,\lfloor K / 2\rfloor$ as in (11). For $n=$ $1, \ldots,\lfloor K / 2\rfloor$ and for each equivalence class $\bar{x} \subset X(K, n)$ w.r.t $\left\{p_{1}, \ldots, p_{K}\right\}$, we have the following upper bounds on $|\bar{x}|$.

(1) If $n=1$, then

$$
|\bar{x}| \leq \begin{cases}r-K, & 1 \geq K-\frac{(1 / \alpha)+1}{2} \\ \frac{1-\alpha}{\ell(K, 1)-\alpha}, & 1<K-\frac{(1 / \alpha)+1}{2}\end{cases}
$$


(2) If $1<n<K-\frac{(1 / \alpha)+1}{2}$, then

$$
|\bar{x}| \leq r+1
$$

(3) If $n=K-\frac{(1 / \alpha)+1}{2}$, then

$$
|\bar{x}| \leq r-K+\left\lfloor 2 \alpha \frac{r-K}{1-\alpha}\right\rfloor .
$$

(4) If $K-\frac{(1 / \alpha)+1}{2}<n<\left\lfloor\frac{K}{2}\right\rfloor$, then

$$
|\bar{x}| \leq s(r, \beta, \gamma), \quad \text { where } \beta=\frac{\alpha-\ell(K, n)}{1-\ell(K, n)} \text { and } \gamma=\frac{-\alpha-\ell(K, n)}{1-\ell(K, n)} .
$$

Corollary 2. Let $X \subset \mathbb{R}^{r}$ be an equiangular set with angle $\alpha$.

(1) If $K_{\alpha}(X)=2<(1 / \alpha)+1$, then $|X| \leq r$.

(2) If $K_{\alpha}(X)=3<(1 / \alpha)+1$, then $|X| \leq 3 r-6$.

Proof. Let $K=K_{\alpha}(X)$. If $K=2$, then the partition (12) becomes $X=\left\{p_{1}, p_{2}\right\} \bigcup X(2,1)$. By Theorem [5, the number of equivalence classes $\bar{x}$ in $X(2,1)$ is $\frac{1}{2}\left(\begin{array}{l}2 \\ 1\end{array}\right)=1$. By Theorem 6 (1), for any $x \in X(2,1),|\bar{x}| \leq r-K=r-2$. So we have $|X|=2+|X(2,1)| \leq 2+r-2=r$.

If $K=3$, then the partition (12) becomes $X=\left\{p_{1}, p_{2}, p_{3}\right\} \bigcup X(3,1)$. By Theorem the number of equivalence classes $\bar{x}$ in $X(3,1)$ is $\left(\begin{array}{l}3 \\ 1\end{array}\right)=3$. By Theorem 6 (1), for any $x \in X(3,1)$, $|\bar{x}| \leq r-K=r-3$. So $|X|=3+|X(3,1)| \leq 3+3 \times(r-3)=3 r-6$.

\subsection{A procedure: piecing together the results}

Once we fix a dimension $r$, the basic procedure to determine an upper bound on the size of an equiangular set $X$ in $\mathbb{R}^{r}$ is as follows:

- From Proposition 1 we know that any maximal set of equiangular lines will either have size $2 r+3$ or have angle $\alpha=1 /(2 L-1)$, where $2 \leq L \leq(1+\sqrt{2 r}) / 2$. This gives us finitely many angles to test.

- For $\alpha=1 / 3$ and $r \geq 15$, we may apply (Lemmens and Seidel, 1973, Theorem 4.5) to determine that $|X| \leq 2(r-1)$.

- For $\alpha<1 / 3$, we consider the possible sizes of a $K$-base in such an $X$, which will be by Propositions 2 and 3 between 2 and $1+1 / \alpha$.

- Then for the $K$-base size $1+1 / \alpha$, we apply Theorem 3 to derive an upper bound, and for each other possible $K$-base size $(<1+1 / \alpha)$, we consider the partition (12) of the remaining elements of $X$ based on the number $(n$ or $K-n)$ of positive inner products with the $K$-base. By Theorem [5, there will be $\lfloor K / 2\rfloor$ such sets of size at most $\left(\begin{array}{l}K \\ n\end{array}\right)$ or $\frac{1}{2}\left(\begin{array}{l}K \\ n\end{array}\right)$.

- By Lemma 5, we then split the partition further into equivalence classes based on with which $K$-base elements an element has positive inner products. We bound the size of each of these equivalence classes by the bounds in Theorem [6.

Below, we illustrate one example to show how to apply the above procedure to compute an upper bound of an equiangular set $X \subset \mathbb{R}^{r}$ for a particular $r$ and $\alpha$. This approach will in particular be used to created Figure 2 (Section 6). 
Example 1. Suppose $r=236$ and $\alpha=1 / 7$. By Propositions 圆 and $K=K_{\alpha}(X)$ is at most $1+1 / \alpha=8$ and at least 2 . Below, we compute an upper bound of $|X|$ for the cases $K=2,3,4,5,6,7$, and 8. Overall, the maximum upper bound in these cases is 15673 and occurs when $K=7$.

- By Corollary圆, if $K=2$, then $|X| \leq r=236$, and if $K=3$, then $|X| \leq 3 r-6=702$.

- If $K=4$, then the partition (12) becomes $X=\left\{p_{1}, p_{2}, p_{3}, p_{4}\right\} \cup \cup_{n=1}^{2} X(4, n)$. For $n=1$, by Theorem [5, the number of equivalence classes $\bar{x}$ in $X(4,1)$ is $\left(\begin{array}{l}4 \\ 1\end{array}\right)=4$. By Theorem 6 (1), for any $x \in X(4,1)$,

$$
|\bar{x}| \leq r-K=232 .
$$

For $n=2$, by Theorem 囵, the number of equivalence classes $\bar{x}$ in $X(4,2)$ is $\frac{1}{2}\left(\begin{array}{l}4 \\ 2\end{array}\right)=3$. Since $K-((1 / \alpha)+1) / 2=4-(5+1) / 2=1<n=2$, we will apply the bound in Theorem 6 (4). To this end, by (6), we calculate $\ell(4,2)=1 / 14$, which implies $\beta=(1 / 7-\ell(4,2)) /(1-\ell(4,2))=$ $1 / 13$, and $\gamma=(-1 / 7-\ell(4,2)) /(1-\ell(4,2))=-3 / 13$, and for any $x \in X(4,2)$,

$$
|\bar{x}| \leq s(r, 1 / 13,-3 / 13) \leq 1832,
$$

where the upper bound 1832 for s $(r, 1 / 13,-3 / 13)$ is computed by Theorem 0 (running SDP). Thus, by the partition,

$$
|X|=4+|X(4,1)|+|X(4,2)| \leq 4+4 \cdot 232+3 \cdot 1832=6428 .
$$

- If $K=5$, then the partition (12) becomes $X=\left\{p_{1}, p_{2}, p_{3}, p_{4}, p_{5}\right\} \cup \cup_{n=1}^{2} X(5, n)$. For $n=1$, by Theorem [5 the number of equivalence classes $\bar{x}$ in $X(5,1)$ is $\left(\begin{array}{l}5 \\ 1\end{array}\right)=5$. By Theorem 6 (1), for any $x \in X(5,1)$,

$$
|\bar{x}| \leq r-K=231 .
$$

For $n=2$, by Theorem [5, the number of equivalence classes $\bar{x}$ in $X(5,2)$ is $\left(\begin{array}{l}5 \\ 2\end{array}\right)=10$. By Theorem [ (4), for any $x \in X(5,2)$,

$$
|\bar{x}| \leq s(r, 1 / 19,-5 / 19) \leq 935 .
$$

So by the partition,

$$
|X|=5+|X(5,1)|+|X(5,2)| \leq 5+5 \cdot 231+10 \cdot 935=10510 .
$$

- If $K=6$, then the partition (12) becomes $X=\left\{p_{1}, p_{2}, p_{3}, p_{4}, p_{5}, p_{6}\right\} \cup \cup_{n=1}^{3} X(6, n)$. For $n=1$, by Theorem [5, the number of equivalence classes $\bar{x}$ in $X(6,1)$ is $\left(\begin{array}{l}6 \\ 1\end{array}\right)=6$. By Theorem [6 (1), for any $x \in X(6,1)$,

$$
|\bar{x}| \leq \frac{1-\alpha}{\ell(K, 1)-\alpha}=\frac{1-1 / 7}{\ell(6,1)-1 / 7}=8 .
$$

For $n=2$, by Theorem 5 , the number of equivalence classes $\bar{x}$ in $X(6,2)$ is $\left(\begin{array}{l}6 \\ 2\end{array}\right)=15$.

By Theorem 6 (3), for any $x \in X(6,2)$,

$$
|\bar{x}| \leq r-K+\left\lfloor 2 \alpha \frac{r-K}{1-\alpha}\right\rfloor=236-6+\left\lfloor 2 \cdot \frac{1}{7} \cdot \frac{236-6}{1-1 / 7}\right\rfloor=306 .
$$


For $n=3$, by Theorem 回, the number of equivalence classes $\bar{x}$ in $X(6,3)$ is $\frac{1}{2}\left(\begin{array}{l}6 \\ 3\end{array}\right)=10$. By Theorem 6 (4), for any $x \in X(6,3)$,

$$
|\bar{x}| \leq s(r, 1 / 25,-7 / 25) \leq 635 .
$$

So by the partition,

$$
|X|=6+|X(6,1)|+|X(6,2)|+|X(6,3)| \leq 6+6 \cdot 8+15 \cdot 306+10 \cdot 635=10994 .
$$

- If $K=7$, then then the partition (12) becomes $X=\left\{p_{1}, p_{2}, p_{3}, p_{4}, p_{5}, p_{6}, p_{7}\right\} \cup \cup_{n=1}^{3} X(7, n)$. For $n=1$, by Theorem [5, the number of equivalence classes $\bar{x}$ in $X(7,1)$ is $\left(\begin{array}{l}7 \\ 1\end{array}\right)=7$. By Theorem 6 (1), for any $x \in X(7,1)$,

$$
|\bar{x}| \leq \frac{1-\alpha}{\ell(K, 1)-\alpha}=\frac{1-1 / 7}{\ell(7,1)-1 / 7}=2 .
$$

For $n=2$, by Theorem [5, the number of equivalence classes $\bar{x}$ in $X(7,2)$ is $\left(\begin{array}{l}7 \\ 2\end{array}\right)=21$. By Theorem [6 (2), for any $x \in X(7,2),|\bar{x}| \leq r+1=237$. For $n=3$, by Theorem [5, the number of equivalence classes $\bar{x}$ in $X(7,3)$ is $\left(\begin{array}{l}7 \\ 3\end{array}\right)=35$. By Theorem $[$ (3), for any $x \in X(7,3)$,

$$
|\bar{x}| \leq r-K+\left\lfloor 2 \alpha \frac{r-K}{1-\alpha}\right\rfloor=236-7+\left\lfloor 2 \cdot \frac{1}{7} \cdot \frac{236-7}{1-1 / 7}\right\rfloor=305 .
$$

So by the partition,

$$
|X|=7+|X(7,1)|+|X(7,2)|+|X(7,3)| \leq 7+7 \cdot 2+21 \cdot 237+35 \cdot 305=15673 .
$$

- If $K=8$, then by Theorem 3 ,

$$
\begin{aligned}
|X| & \leq K+\frac{1}{2}\left(\begin{array}{c}
K \\
K / 2
\end{array}\right)\left(r-K+1+\left\lfloor 2 \alpha \frac{r-K+1}{1-\alpha}\right\rfloor\right) \\
& =8+\frac{1}{2}\left(\begin{array}{l}
8 \\
4
\end{array}\right)\left(236-8+1+\left\lfloor 2 \cdot \frac{1}{7} \cdot \frac{236-8+1}{1-1 / 7}\right\rfloor\right)=10683 .
\end{aligned}
$$

\section{Relative bound for $\alpha=1 / 5$}

Applying the procedure in the previous section as presented in Example 1. one can derive an upper bound for equiangular sets with any angle. But this upper bound might not be optimal. One reason is that when two different equivalence classes are not empty, each equivalence class might not really be filled with the number of vectors given in Theorem 3 or Theorem 6 (evidence of this can be seen in (Lemmens and Seidel, 1973, Theorem 4.3)). In this section, we use this observation to prove an upper bound when $\alpha=1 / 5$ (Theorem 7). The basic idea is to apply the procedure but then to analyze further when two equivalence classes are not empty (see the proofs of Lemmas 7 8 in Appendices CD . One could perform similar analysis for any smaller angles (such as $\alpha=1 / 7,1 / 9, \ldots)$, but it would be a very technical task.

Lemma 6. For any $r \in \mathbb{N}$ and $0 \leq \alpha<1$, there exist at least $r$ vectors in $\mathbb{R}^{r}$ with pairwise inner product $\alpha$. 
Proof. Consider the $r \times r$ real symmetric matrix $G=(1-\alpha) I_{r}+\alpha J_{r}$. By Lemma 4 , $G$ has two distinct eigenvalues

$$
\lambda_{1}=1+(r-1) \alpha, \quad \lambda_{2}=1-\alpha .
$$

Both eigenvalues are positive. So $G$ is positive definite. By the well-known Cholesky decomposition, there exists a unique $r \times r$ lower triangular matrix $L$ such that $G=L L^{T}$. Suppose the column vectors of $L$ are $x_{1}, \ldots, x_{r}$. Then $G$ is the Gramian matrix of $x_{1}, \ldots, x_{r}$.

Geometrically, we may think of "pushing" vectors in an orthonormal basis for $\mathbb{R}^{r}$ towards each other until the desired angle is achieved.

Corollary 3. For any positive integer $r$, if $s(r, 1 / 13,-5 / 13)$ denotes the maximum cardinality of a spherical two-distance set in $\mathbb{R}^{r}$ w.r.t. $1 / 13$ and $-5 / 13$, then

$$
s(r, 1 / 13,-5 / 13) \geq r .
$$

Proof. We know from Lemma 6 that there are at least $r$ vectors with inner product 1/13.

For the cases $K_{1 / 5}(X)=4,5$, we present Lemmas 78 below. The proofs of the two lemmas are given in Appendices $\mathrm{C}$ and $\mathrm{D}$, respectively.

Lemma 7. Let $X \subset \mathbb{R}^{r}$ be an equiangular set with angle $1 / 5$. If $K_{1 / 5}(X)=4$, then

$$
|X| \leq 148+3 \cdot s(r, 1 / 13,-5 / 13) .
$$

Lemma 8. Let $X \subset \mathbb{R}^{r}$ be an equiangular set with angle $1 / 5$. If $K_{1 / 5}(X)=5$, then

$$
|X| \leq \begin{cases}290, & 23 \leq r \leq 185 \\ r+15+\lfloor(r-5) / 2\rfloor, & r \geq 185\end{cases}
$$

Theorem 7. Let $X \subset \mathbb{R}^{r}$ be an equiangular set with angle $1 / 5$. If $r>60$, then

$$
|X| \leq 148+3 \cdot s(r, 1 / 13,-5 / 13) .
$$

Proof. By Proposition 2 $K_{1 / 5}(X)$ is at most $(1 / 5)^{-1}+1=6$, and by Proposition $3 K_{1 / 5}(X)$ is at least 2 . So it is only possible for $K_{1 / 5}(X)$ to be $2,3,4,5$, or 6 .

- By Corollary 2, if $K_{1 / 5}(X)=2$, then $|X| \leq r$, and if $K_{1 / 5}(X)=3$, then $|X| \leq 3 r-6$.

- If $K_{1 / 5}(X)=4$, then by Lemma $7|X| \leq 148+3 \cdot s(r, 1 / 13,-5 / 13)$.

- If $K_{1 / 5}(X)=5$, then by Lemma $8|X| \leq \max (290, r+15+\lfloor(r-5) / 2\rfloor)$.

- If $K_{1 / 5}(X)=6$, then by Theorem $4,|X| \leq \max (276, r+1+\lfloor(r-5) / 2\rfloor)$.

Overall, $|X|$ should be bounded by the maximum upper bound in the above cases. Note $r \leq 3 r-6$ when $r \geq 3$. So

$$
\begin{aligned}
|X| & \leq \max (r, 3 r-6,148+3 \cdot s(r, 1 / 13,-5 / 13), 290, r+15+\lfloor(r-5) / 2\rfloor, 276, r+1+\lfloor(r-5) / 2\rfloor) \\
& =\max (3 r-6,148+3 \cdot s(r, 1 / 13,-5 / 13), 290, r+15+\lfloor(r-5) / 2\rfloor) .
\end{aligned}
$$


By Corollary $3 s(r, 1 / 13,-5 / 13) \geq r$. So

$$
148+3 \cdot s(r, 1 / 13,-5 / 13) \geq 148+3 r>\max \{3 r-6, r+15+\lfloor(r-5) / 2\rfloor\} .
$$

and hence

$$
|X| \leq \max (148+3 \cdot s(r, 1 / 13,-5 / 13), 290) .
$$

If $r>60$, then we further have

$$
148+3 \cdot s(r, 1 / 13,-5 / 13) \geq 148+3 r>148+3 \times 60>290 .
$$

So if $r>60$,

$$
|X| \leq 148+3 \cdot s(r, 1 / 13,-5 / 13) .
$$

Lemma 9. Glazyrin and $Y u$, 2016, Corollary 4) If $Y$ is a spherical two-distance set in $\mathbb{R}^{r}$ w.r.t. $\alpha$ and $\beta$, then

$$
|Y| \leq \frac{r+2}{1-\frac{r-1}{r(1-\alpha)(1-\beta)}}
$$

Corollary 4. For a given equiangular set $X \subset \mathbb{R}^{r}$ with angle $1 / 5$ if $r>60$, then

$$
|X| \leq 148+\frac{648 r(r+2)}{47 r+169}
$$

Proof. By Theorem 7 and Lemma 9 (Glazyrin and Yu, 2016, Corollary 4),

$$
|X| \leq 148+3 \cdot s(r, 1 / 13,-5 / 13) \leq 148+3 \frac{r+2}{1-\frac{r-1}{r(1-1 / 13)(1+5 / 13)}}=148+\frac{648 r(r+2)}{47 r+169} .
$$

\begin{tabular}{|c|c|c|c|c|c|}
\hline dimension & Theorem 7 & $\begin{array}{l}\text { Corollary } 4 \\
4\end{array}$ & dimension & Theorem 7 & Corollary 4 \\
\hline$r$ & $148+3 \cdot \mathrm{s}\left(r, \frac{1}{13},-\frac{5}{13}\right)$ & $148+\frac{648 r(r+2)}{47 r+169}$ & $r$ & $148+3 \cdot \mathrm{s}\left(r, \frac{1}{13},-\frac{5}{13}\right)$ & $148+\frac{648 r(r+2)}{47 r+169}$ \\
\hline 61 & 586 & 968 & 62 & 595 & 982 \\
\hline 63 & 604 & 995 & 64 & 616 & 1009 \\
\hline 65 & 625 & 1023 & 66 & 637 & 1037 \\
\hline 67 & 646 & 1050 & 68 & 658 & 1064 \\
\hline 69 & 667 & 1078 & 70 & 679 & 1092 \\
\hline 71 & 691 & 1105 & 72 & 703 & 1119 \\
\hline 73 & 715 & 1133 & 74 & 727 & 1147 \\
\hline 75 & 739 & 1161 & 76 & 751 & 1174 \\
\hline 77 & 763 & 1188 & 78 & 775 & 1202 \\
\hline 79 & 787 & 1216 & 80 & 799 & 1229 \\
\hline 81 & 814 & 1243 & 82 & 826 & 1257 \\
\hline 83 & 841 & 1271 & 84 & 853 & 1285 \\
\hline 85 & 868 & 1298 & 86 & 883 & 1312 \\
\hline 87 & 898 & 1326 & 88 & 910 & 1340 \\
\hline 89 & 925 & 1353 & 90 & 943 & 1367 \\
\hline 91 & 958 & 1381 & 92 & 973 & 1395 \\
\hline
\end{tabular}

Remark 2. We have a few remarks about Theorem 7 and Corollary 4

(i) Note $s(r, 1 / 13,-5 / 13)$ in the bound given in Theorem 7 can be computed bounded by Theorem 圆 (running SDP tool). Comparing this to the explicit bounds given in Corollary 4 , the bounds computed by SDP according to Theorem 7 are smaller for $61 \leq r \leq 132$, see the Table Q However, for $r>132$, Corollary 4 gives the smaller bounds. 


\begin{tabular}{|c|c|c|c|c|c|}
\hline 93 & 988 & 1409 & 94 & 1006 & 1422 \\
\hline 95 & 1021 & 1436 & 96 & 1039 & 1450 \\
\hline 97 & 1057 & 1464 & 98 & 1072 & 1477 \\
\hline 99 & 1090 & 1491 & 100 & 1108 & 1505 \\
\hline 101 & 1126 & 1519 & 102 & 1147 & 1533 \\
\hline 103 & 1165 & 1546 & 104 & 1186 & 1560 \\
\hline 105 & 1204 & 1574 & 106 & 1225 & 1588 \\
\hline 107 & 1246 & 1601 & 108 & 1267 & 1615 \\
\hline 109 & 1288 & 1629 & 110 & 1309 & 1643 \\
\hline 111 & 1333 & 1657 & 112 & 1354 & 1670 \\
\hline 113 & 1378 & 1684 & 114 & 1402 & 1698 \\
\hline 115 & 1426 & 1712 & 116 & 1453 & 1725 \\
\hline 117 & 1477 & 1739 & 118 & 1504 & 1753 \\
\hline 119 & 1531 & 1767 & 120 & 1558 & 1781 \\
\hline 121 & 1585 & 1794 & 122 & 1612 & 1808 \\
\hline 123 & 1642 & 1822 & 124 & 1672 & 1836 \\
\hline 125 & 1702 & 1850 & 126 & 1732 & 1863 \\
\hline 127 & 1765 & 1877 & 128 & 1798 & 1891 \\
\hline 129 & 1831 & 1905 & 130 & 1867 & 1918 \\
\hline 131 & 1900 & 1932 & 132 & 1936 & 1946 \\
\hline
\end{tabular}


(ii) Notice also that the relative bound given in Corollary 4 is asymptotically $\frac{648}{17} r \sim 13.8 r$. For any $r>60$, this upper bound is smaller and thus better than Glazyrin and Yu, 2016, Theorem 3)

$$
r\left(\frac{2}{3} a^{2}+\frac{4}{7}\right)+\left.2\right|_{a=5}=\frac{362}{21} r+2 \sim 17.2 r .
$$

(iii) In (Neumaier, 1989), it is proven that there exists a large integer $N$ such that for any $r \geq N$, the relative bound for $\alpha=1 / 5$ is

$$
r+1+\lfloor(r-5) / 2\rfloor \sim 1.5 r
$$

The $N$ stated in (Neumaien, 1989, p. 155) is claimed to be between 2486 and 45374 without proof. However, there is still a big gap between the above $1.5 \mathrm{r}$ and the $13.8 \mathrm{r}$ in Corollary 4. It would be interesting to shorten the gap for $61<r<N$.

\section{Computational Result and Conjecture}

We are now prepared to show upper bounds for equiangular sets for $44 \leq r \leq 400$.

Theorem 8. For $44 \leq r \leq 400$, let $m$ be the largest positive integer such that $(2 m+1)^{2} \leq r+2$. Then an upper bound of the maximum number of equiangular lines in $\mathbb{R}^{r}$ is

$$
\begin{cases}\frac{4 r(m+1)(m+2)}{(2 m+3)^{2}-r}, & r=44,45,46,76,77,78,117,118,166,222,286,358 \\ \frac{\left((2 m+1)^{2}-2\right)\left((2 m+1)^{2}-1\right)}{2}, & \text { other } r \text { between } 44 \text { and } 400,\end{cases}
$$

and if the upper bound in (13) can be attained, then the relative angle is

$$
\begin{cases}\frac{1}{2 m+3}, & r=44,45,46,76,77,78,117,118,166,222,286,358 \\ \frac{1}{2 m+1}, & \text { other } r \text { between } 44 \text { and } 400 .\end{cases}
$$

Proof. For any $44 \leq r \leq 400$, suppose $L$ is the largest positive integer such that $(2 L-1)^{2} \leq 2 r$. For each $r$, by Proposition 1. we compute the relative bounds for each $\alpha=1 / 5,1 / 7, \ldots, 1 /(2 L-1)$ and then pick up the maximum. We summarize the computational results in Table 3 (see the complete computational results online (King and Tang, 2018))

More specifically, for $\alpha=1 / 5$, we compute three upper bounds by Thoerem 2, Theorem 7 , and Corollary 4, respectively, and then we pick the smaller one of these three upper bounds. For $\alpha=1 / 7$, we compute two upper bounds by Theorem[2 and the procedure in Section 3.5 (Example11), respectively, and then we pick the smaller one of these two upper bounds. For $\alpha=1 / 9, \ldots, 1 /(2 L-$ 1), we compute the SDP bound according to Theorem 2 One can check Table 3 is equivalent to (13 14).

We remark that more computations can be carried out for $r>400$. For those large $r$ 's, in order to get a non-trivial bound (less than Gerzon's bound) for $\alpha \leq 1 / 9$, one can apply the procedure like what we have done for $\alpha=1 / 7$ in Example 1 According to Table 1 Theorem 8 , and our further experiments, we propose a conjecture below. 
Table 3: Upper bound of equiangular lines in $\mathbb{R}^{r}$ for $44 \leq r \leq 400$

\begin{tabular}{c||ccccccc}
$r$ & 44 & 45 & 46 & $47-75$ & 76 & 77 & \\
$|X| \leq$ & 422 & 540 & 736 & 1128 & 1216 & 1540 & \\
$\underset{\alpha}{\operatorname{argmax}}|X|$ & $\frac{1}{7}$ & $\frac{1}{7}$ & $\frac{1}{7}$ & $\frac{1}{7}$ & $\frac{1}{9}$ & $\frac{1}{9}$ & \\
\hline \hline$r$ & 78 & $79-116$ & 117 & 118 & $119-165$ & 166 & \\
$|X| \leq$ & 2080 & 3160 & 3510 & 4720 & 7140 & 9296 & \\
$\operatorname{argmax}|X|$ & $\frac{1}{9}$ & $\frac{1}{9}$ & $\frac{1}{11}$ & $\frac{1}{11}$ & $\frac{1}{11}$ & $\frac{1}{13}$ & \\
\hline \hline$r$ & $167-221$ & 222 & $223-285$ & 286 & $287-357$ & 358 & $359-400$ \\
$|X| \leq$ & 14028 & 16576 & 24976 & 27456 & 41328 & 42960 & 64620 \\
$\underset{\alpha}{\operatorname{argmax}}|X|$ & $\frac{1}{13}$ & $\frac{1}{15}$ & $\frac{1}{15}$ & $\frac{1}{17}$ & $\frac{1}{17}$ & $\frac{1}{19}$ & $\frac{1}{19}$
\end{tabular}

Conjecture 1. For any $r$, if $m$ is the largest positive integer such that $(2 m+1)^{2} \leq r+2$, then an upper bound of maximum number of equiangular lines in $\mathbb{R}^{r}$ is either $\frac{4 r(m+1)(m+2)}{(2 m+3)^{2}-r}$ or $\frac{\left((2 m+1)^{2}-2\right)\left((2 m+1)^{2}-1\right)}{2}$.

\section{Experiments}

We compare the relative bounds for $\alpha=1 / 5$ given by Corollary 4 and the basic SDP method (Theorem 2) without pillar decomposition (Barg and Yu, 2014) in Figure11. For each $r$ between 44 and 400, we compute the two upper bounds by Corollary 4 and Theorem 2 for $\alpha=1 / 5$, respectively. In Figure 1 we mark by blue plusses "+" the computed upper bound according to Corollary 4 and we mark the bounds due to Theorem 2 (SDP bounds without pillar decomposition) by red stars "^". We also draw Gerzon's bound as a black curve.

Similarly, in the Figure 2, we compare the relative bounds for $\alpha=1 / 7$ computed by the procedure shown in Example 1 and the SDP method without pillar decomposition.

In our experiments, we solve SDP by the Matlab software CVX 3.0 beta Grant and Bovd, 2014, 2008). There are many SDP solvers provided by CVX 3.0 beta and the computational results presented in this paper are computed by sdpt3 (Toh et al., 1999; Tutuncu et al., 2003). The computation is carried out by a $3.20 \mathrm{GHz} \operatorname{Interl}(\mathrm{R})$ Core(TM) i5-4460 processor under x86_64 GNU/Linux. Our Matlab version is R2016a. The code used for the calculations in this paper may be found at (King and Tang, 2018, code). The output of sdpt3 is a floating number. We take the nearest integer of this floating number.

We see in Figures 1 2 for $44 \leq r \leq 400$ that the "red" SDP bound is smaller when $r$ is small, it increases dramatically at some $r$, and it eventually goes beyond the Gerzon's bound. Further, our "blue" bound is larger when $r$ is small, but it increases slower, and when $r$ is sufficiently large, it always gives non-trivial upper bound which is much smaller than either the SDP bound or the Gerzon's bound. Some further comments are as follows. See (King and Tang, 2018, table.pdf) for the concrete data.

(Comment 1). Concerning Figure 1 when $44 \leq r \leq 93$, the SDP bound without pillar decomposition is smaller than the bound in Theorem 7 . When $94 \leq r \leq 400$, the bound in Theorem 7 is smaller (generally much smaller) than the SDP bound. One can check the concrete 
Figure 1: Comparing Corollary 4 and Theorem 2

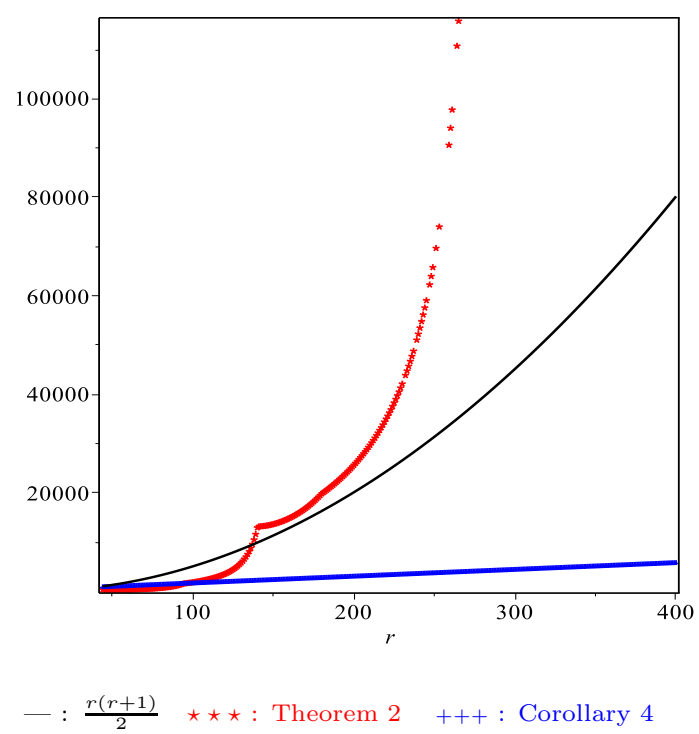

Figure 2: Comparing Example 1 and SDP bound

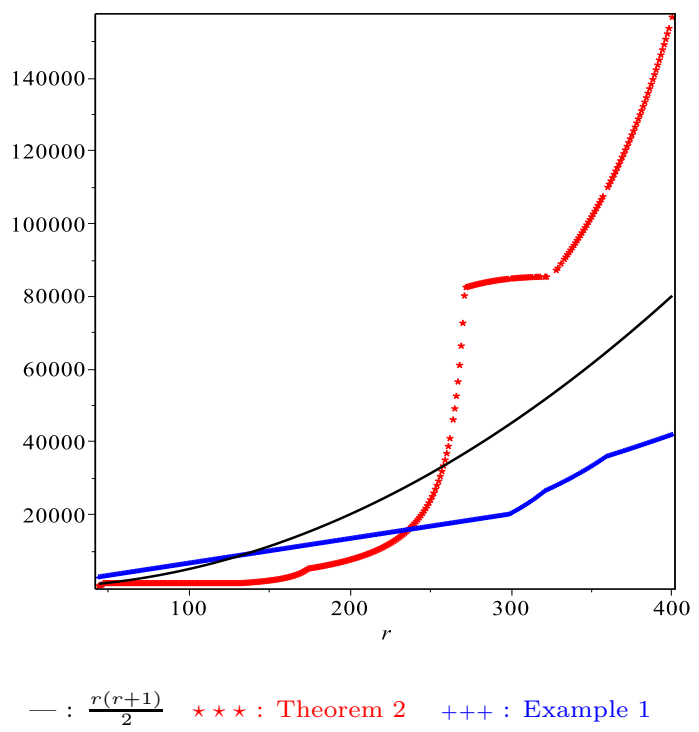

bounds for $r \geq 94$ in King and Tang, 2018, Table.pdf) and compare them with the SDP bounds shown in the red parentheses. The SDP bounds in the red parentheses which we computed are the same as the data shown in (Barg and Yu, 2014), which is to be expected. As an example of Theorem 7 outperforming SDP, the bound given by Theorem 7 for $r=137$ is 2015 while the SDP bound is 9528 . When $137 \leq r \leq 400$, the SDP bound is greater (eventually much greater) than Gerzon's bound, which is consistent with the results obtained in (Barg and Yu, 2014). On the other hand, for any $44 \leq r \leq 400$, the bound in Theorem 7 is smaller than Gerzon's bound. For a range of larger $r$, for instance $r=266-400$ and some discrete $r$ such as 231, 238 and so on, sdpt3 failed to compute the SDP bound. That is the reason why the red markers in Figure 1 are not as continuous as the blue markers.

(Comment 2). Similarly, we note for Figure 2 that when $44 \leq r \leq 235$, the SDP bound is smaller. When $235 \leq r \leq 400$, the "blue" bound is smaller (generally much smaller) than the SDP bound. When $259 \leq r \leq 400$, the SDP bound is greater (eventually much greater) than Gerzon's bound. For some large $r$, sdpt3 failed to compute the SDP bound.

In conclusion, practically, for $\alpha=1 / 5$ or $1 / 7$ and for any $r$, we can compute both upper bounds for $s_{\alpha}(r)$ : the SDP bound and the "blue" bound, and then pick up the smaller one.

An extra remark is that for $\alpha<1 / 7$, experiments show that the SDP bounds are always no greater than the Gerzon's bound for $44 \leq r \leq 400$. See the SDP bounds in the columns " $1 / 9$ ", $\ldots$, "1/27" in (King and Tang, 2018, Table.pdf). A possible reason is that 400 is not large enough for the SDP bound $(r \leq 400, \alpha<1 / 7)$ to go beyond the Gerzon's bound.

\section{Acknowledgments}

We are particularly indebted to Bernd Sturmfels for introducing the authors to each other. We thank Toh Kim Chuan for his nice answers to our questions about sdpt3. We are very appreciative 
of Wei-Hsuan Yu and Gary Greaves, who pointed out the newest bounds on $s(17)-s(20)$. Finally, we thank Eva Maria Feichtner for her helpful comments on this work. The authors were supported in part by the Zentrum für Forschungsförderung der Uni Bremen Explorationsprojekt "Hilbert Space Frames and Algebraic Geometry."

\section{References}

\section{References}

Azarija, J., Marc, T., 2016. There is no (95, 40, 12, 20) strongly regular graph, http://arxiv.org/abs/1603.02032.

Bachoc, C., Vallentin, F., 2008. New upper bounds for kissing numbers from semidefinite programming. J. Amer. Math. Soc. 21, 909-924.

Balla, I., Dräxler, F., Keevash, P., Sudakov, B., 2018. Equiangular lines and spherical codes in Euclidean space. Invent. Math. 211 (1), 179-212.

URL https://doi.org/10.1007/s00222-017-0746-0

Barg, A., Yu, W.-H., 2013. New bounds for spherical two-distance sets. Experiment. Math. 22 (2), 187-194.

Barg, A., Yu, W.-H., 2014. New bounds for equiangular lines. In: Barg, A., Musin, O. R. (Eds.), Discrete Geometry and Algebraic Combinatorics. Vol. 625 of Contemporary Mathematics. Amer. Math. Soc. Providence, pp. 111-121.

Benedetto, J. J., Kolesar, J. D., 2006. Geometric properties of Grassmannian frames for $\mathbb{R}^{2}$ and $\mathbb{R}^{3}$. EURASIP Journal on Applied Signal Processing 1, 1-17.

Bodmann, B. G., 2007. Optimal linear transmission by loss-insensitive packet encoding. Appl. Comput. Harmon. Anal. $22(3), 274-285$.

Bruckstein, A. M., Donoho, D. L., Elad, M., 2009. From sparse solutions of systems of equations to sparse modeling of signals and images. SIAM Rev. 51 (1), 34-81.

Conway, J. H., Hardin, R. H., Sloane, N. J. A., 1996. Packing lines, planes, etc.: packings in Grassmannian spaces. Experiment. Math. 5 (2), 139-159.

Coxeter, H. S. M., 1963. Regular polytopes, 2nd Edition. Macmillan, New York.

Delsarte, P., Goethals, J. M., Seidel, J. J., 1977. Spherical codes and designs. Geometriae Dedicata 6 (3), 363-388.

Dhillon, I. S., Heath, J. R., Strohmer, T., Tropp, J. A., 2008. Constructing packings in Grassmannian manifolds via alternating projection. Experiment. Math. 17 (1), 9-35.

Ding, C., Feng, T., 2007. A generic construction of complex codebooks meeting the Welch bound. IEEE Trans. Inform. Theory 53, 4245-4250.

Donoho, D. L., Elad, M., 2003. Optimally sparse representation in general (nonorthogonal) dictionaries via $l^{1}$ minimization. Proc. Natl. Acad. Sci. USA 100 (5), 2197-2202 (electronic).

Fickus, M., Jasper, J., King, E. J., Mixon, D. G., 2017. Equiangular tight frames that contain regular simplices. URL https://arxiv.org/abs/1711.07081

Fickus, M., Mixon, D. G., Tremain, J. C., 2012. Steiner equiangular tight frames. Linear Algebra Appl. 436, 10141027.

Glazyrin, A., Yu, W.-H., 2016. Upper bounds for s-distance sets and equiangular lines, arXiv:1611.09479.

Godsil, C., Royle, G., 2001. Algebraic graph theory. Vol. 207 of Graduate Texts in Mathematics. Springer-Verlag, New York.

URL https://doi.org/10.1007/978-1-4613-0163-9 
Gosselin, S., 2004. Regular Two-Graphs and Equiangular Lines. Master's thesis, University of Waterloo, Waterloo, Ontario, Canada.

Grant, M., Boyd, S., 2008. Graph implementations for nonsmooth convex programs. In: Blondel, V., Boyd, S., Kimura, H. (Eds.), Recent Advances in Learning and Control. Springer-Verlag Limited, pp. 95-110.

Grant, M., Boyd, S., mar 2014. CVX: Matlab software for disciplined convex programming, version 2.1. http://cvxr.com/cvx

Greaves, G., Koolen, J. H., Munemasa, A., Szöll" osi, F., 2016. Equiangular lines in Euclidean spaces. J. Combin. Theory Ser. A 138, 208-235. URL https://doi.org/10.1016/j.jcta.2015.09.008

Greaves, G. R. W., 2018. Equiangular line systems and switching classes containing regular graphs. Linear Algebra Appl. 536, 31-51. URL https://doi.org/10.1016/j.laa.2017.09.008

Greaves, G. R. W., Yatsyna, P., 2018. A modular characterisation of the characteristic polynomial of a Seidel matrix, in preperation.

Haantjes, J., 1948. Equilateral point-sets in elliptic two- and three-dimensional spaces. Nieuw Arch. Wiskunde (2) $22,355-362$.

Holmes, R. B., Paulsen, V. I., 2004. Optimal frames for erasures. Linear Algebra Appl. 377, 31-51.

Horn, R. A., Johnson, C. R., 2012. Matrix Analysis, 2nd Edition. Cambridge University Press.

King, E., Tang, X., Febarury 2018. Supplementary materials: Computational results and code. https://sites.google.com/site/rootclassification/supplementary-materials/el

Larman, D. G., Rogers, C. A., Seidel, J. J., 1977. On two-distance sets in Euclidean space. Bulletin of the London Mathematical Society 9 (3), 261-267.

Lemmens, P. W., Seidel, J. J., 1973. Equiangular lines. Journal of Algebra 24 (3), 494-512.

Musin, O. R., 2009. Spherical two-distance sets. J. Combin. Theory Ser. A 116 (4), 988-995.

Neumaier, A., 1989. Graph representations, two-distance sets, and equiangular lines. Linear Algebra Appl. 114, $141-156$.

Strohmer, T., Heath, Jr., R. W., 2003. Grassmannian frames with applications to coding and communication. Appl. Comput. Harmon. Anal. 14 (3), 257-275.

Sustik, M. A., Tropp, J. A., Dhillon, I. S., Heath Jr, R. W., 2007. On the existence of equiangular tight frames. Linear Algebra Appl. 426, 619-635.

Szöllösi, F., Sep 2017. A remark on a construction of d.s. asche. Discrete Comput. Geom. URL https://doi.org/10.1007/s00454-017-9933-4

Toh, K. C., Todd, M. J., Tutuncu, R. H., 1999. a matlab software package for semidefinite programming. Optimization Methods and Software 11, 545-581.

Tutuncu, R. H., Toh, K. C., Todd, M. J., 2003. Solving semidefinite-quadratic-linear programs using sdpt3. Mathematical Programming Ser. B 95, 189-217.

Van Lint, J. H., Seidel, J. J., 1966. Equilateral point sets in elliptic geometry. Indagationes Mathematicae, Proc. Koninkl. Ned. Akad. Wetenschap. Ser. A 69 (3), 335-34.

Waldron, S., 2009. On the construction of equiangular frames from graphs. Linear Algebra Appl. 431, $2228-2242$.

Xia, P., Zhou, S., Giannakis, G. B., 2005. Achieving the Welch bound with difference sets. IEEE Trans. Inform. Theory 51, 1900-1907. 
Yu, W.-H., 2014. Spherical two-distance sets and related topics in harmonic analysis. Ph.D. thesis, University of Maryland College Park.

Yu, W.-H., 2015. There are no 76 equiangular lines in $\mathrm{R}^{19}$, http://arxiv.org/abs/1511.08569.

\section{Appendices}

\section{A. Proof of Theorem 3}

In Appendix $\mathrm{A}$, let $X \subset \mathbb{R}^{r}$ be an equiangular set with angle $\alpha$, where $1 / \alpha$ is an odd number greater or equal to 3 . Let $K=K_{\alpha}(X)$. For any $K$-base $\left\{p_{1}, \ldots, p_{K}\right\} \subset X$ such that $\left\langle p_{i}, p_{j}\right\rangle=-\alpha$ $(\forall 1 \leq i<j \leq K)$, let $P$ be the linear subspace of $\mathbb{R}^{r}$ spanned by $p_{1}, \ldots, p_{K}$ and let $P^{\perp}$ be the orthogonal complement of $P$ in $\mathbb{R}^{r}$. For any $x \in X \backslash\left\{p_{1}, \ldots, p_{K}\right\}$, the following lemma originally comes from (Lemmens and Seidel, 1973, Section 4). The arguments to prove the following lemma are so similar for both cases $K=(1 / \alpha)+1$ and $K<(1 / \alpha)+1$ that we combine them, even though $K<(1 / \alpha)+1$ applies to Appendix B

Lemma 10. Let $X$ be an equiangular set in $\mathbb{R}^{r}$ with $\alpha$. We set $K=K_{\alpha}(X)$. Then for each equivalence class $\bar{x} \subset X$ w.r.t any fixed $K$-base $\left\{p_{1}, \ldots, p_{K}\right\}$,

$$
|\bar{x}| \leq \begin{cases}r-K+1+\left\lfloor 2 \alpha \frac{r-K+1}{1-\alpha}\right\rfloor, & K=(1 / \alpha)+1 \\ s(r, \beta, \gamma), & K<(1 / \alpha)+1, \quad x \in X(K, n), \quad n=1, \ldots,\lfloor K / 2\rfloor\end{cases}
$$

where $X(K, n)$ is defined as in (11), $\beta=(\alpha-\ell(K, n)) /(1-\ell(K, n))$ and $\gamma=(-\alpha-\ell(K, n)) /(1-$ $\ell(K, n))$.

Proof. Let $\bar{x}=\left\{x_{1}, \ldots, x_{s}\right\}$. By Corollary 1, we can assume that for each $i=1, \ldots, s$,

$$
x_{i}=h+c_{i}, \quad \text { where } h \in P \text { and } c_{i} \in P^{\perp} .
$$

Then

$$
G(\bar{x})=\langle h, h\rangle J+G\left(c_{1}, \ldots, c_{s}\right) .
$$

Note the norm squared of $c_{i}$ is $\left\langle c_{i}, c_{i}\right\rangle=\left\langle x_{i}, x_{i}\right\rangle-\langle h, h\rangle=1-\langle h, h\rangle$. By Proposition 4 , if $K=$ $(1 / \alpha)+1$, then $\langle h, h\rangle=\alpha<1$ (note $0<\alpha<1$ by Definition 10), and if $K<(1 / \alpha)+1$, then $\langle h, h\rangle<1$ (see Formula (77)). So we always have $1-\langle h, h\rangle \neq 0$. If we normalize the vectors $c_{1}, \ldots, c_{s}$ as $c_{1}^{\prime}, \ldots, c_{s}^{\prime}$, then the Gramian matrix of $c_{1}^{\prime}, \ldots, c_{s}^{\prime}$ is

$$
G\left(c_{1}^{\prime}, \ldots, c_{s}^{\prime}\right)=\frac{G\left(c_{1}, \ldots, c_{s}\right)}{1-\langle h, h\rangle}=\frac{G(\bar{x})-\langle h, h\rangle J}{1-\langle h, h\rangle} .
$$

Obviously, this matrix has 1's along the diagonal. Note $G(\bar{x})$ has $\alpha$ or $-\alpha$ as the off-diagonal entries. So $G\left(c_{1}^{\prime}, \ldots, c_{s}^{\prime}\right)$ has two different numbers $(\alpha-\langle h, h\rangle) /(1-\langle h, h\rangle)$ or $(-\alpha-\langle h, h\rangle) /(1-\langle h, h\rangle)$ as the off-diagonal entries. So $\left\{c_{1}^{\prime}, \ldots, c_{s}^{\prime}\right\}$ is a spherical two-distance set such that

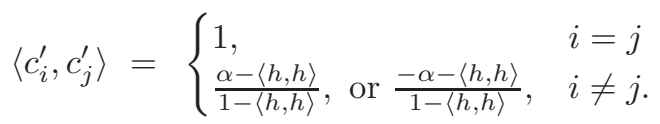


Thus $\bar{x} \leq s(r, \beta, \gamma)$, with $\beta=(\alpha-\langle h, h\rangle) /(1-\langle h, h\rangle)$ and $\gamma=(-\alpha-\langle h, h\rangle) /(1-\langle h, h\rangle)$. The bounds follow from the values of $\langle h, h\rangle$ in Proposition 4

$$
\langle h, h\rangle= \begin{cases}\alpha, & K=(1 / \alpha)+1 \\ \ell(K, n), & K<(1 / \alpha)+1, x \in X(K, n), n=1, \ldots,\lfloor K / 2\rfloor\end{cases}
$$

and, for $K=(1 / \alpha)+1$, since $\beta=0$ and $\gamma=-2 \alpha /(1-\alpha)$, it follows from (Lemmens and Seidel, 1973, Theorem 4.1) that $s(r, 0,-2 \alpha /(1-\alpha)) \leq r-K+1+\lfloor 2 \alpha(r-K+1) /(1-\alpha)\rfloor$.

Lemma 11. For any $x \in X \backslash\left\{p_{1}, \ldots, p_{K}\right\}$, suppose

$$
\left(\left\langle x, p_{1}\right\rangle, \ldots,\left\langle x, p_{K}\right\rangle\right)^{\top}=\alpha \cdot\left(\epsilon^{(1)}, \ldots, \epsilon^{(K)}\right)^{\top}, \text { where } \epsilon^{(i)}= \pm 1 .
$$

If $K=(1 / \alpha)+1$, then $\epsilon^{(1)}+\cdots+\epsilon^{(K)}=0$.

Proof. By Proposition 2 $p_{1}+\cdots+p_{K}=0$ since $K=(1 / \alpha)+1$. Suppose we decompose $x$ in $P$ and $P^{\perp}$ as

$$
x=h+c, \text { where } h \in P \text { and } c \in P^{\perp} .
$$

Then $\left\langle h, p_{1}+\cdots+p_{K}\right\rangle=0$. On the other hand, we have

$$
\left\langle h, p_{1}+\cdots+p_{K}\right\rangle=\left\langle h, p_{1}\right\rangle+\cdots+\left\langle h, p_{K}\right\rangle=\left\langle x, p_{1}\right\rangle+\cdots+\left\langle x, p_{K}\right\rangle=\alpha\left(\epsilon^{(1)}+\ldots+\epsilon^{(K)}\right) .
$$

Since $\alpha \neq 0, \epsilon^{(1)}+\ldots+\epsilon^{(K)}=0$.

Proof of Theorem 3 . Note $K=(1 / \alpha)+1$ is even since $1 / \alpha$ is odd. By Lemma 11 and Lemma 5 , there are $\frac{1}{2}\left(\begin{array}{c}K \\ K / 2\end{array}\right)$ equivalence classes $\bar{x}$ in $X$. More specifically, we index all the $(1,-1)$-vectors in $\mathbb{R}^{K}$ with exactly $K / 21$-coordinates and the first coordinate 1 as

$$
\epsilon_{m}, \quad m=1, \ldots, \frac{1}{2}\left(\begin{array}{c}
K \\
K / 2
\end{array}\right)
$$

and for each $\epsilon_{m}$, we define

$$
\bar{x}_{m}=\left\{x \in X \backslash\left\{p_{1}, \ldots, p_{K}\right\} \mid\left(\left\langle x, p_{1}\right\rangle, \ldots,\left\langle x, p_{K}\right\rangle\right)= \pm \alpha \cdot \epsilon_{m}\right\} .
$$

Then we have a partition of $X$

$$
X=\left\{p_{1}, \ldots, p_{K}\right\} \bigcup \bigcup_{m=1}^{\frac{1}{2}\left(\begin{array}{c}
K \\
K / 2
\end{array}\right)} \bar{x}_{m}
$$

By the partition (15) and Lemma 10, we have

$$
|X| \leq K+\frac{1}{2}\left(\begin{array}{c}
K \\
K / 2
\end{array}\right)\left(r-K+1+\left\lfloor 2 \alpha \frac{r-K+1}{1-\alpha}\right\rfloor\right) .
$$




\section{B. Proof of Theorem 6}

In Appendix B, let $X \subset \mathbb{R}^{r}$ be an equiangular set with angle $\alpha$ and let $K$ be $K=K_{\alpha}(X)<$ $(1 / \alpha)+1$. Again, we fix a $K$-base $\left\{p_{1}, \ldots, p_{K}\right\} \subset X$ such that $\left\langle p_{i}, p_{j}\right\rangle=-\alpha(\forall 1 \leq i<j \leq K)$. Let $P$ be the linear subspace of $\mathbb{R}^{r}$ spanned by $p_{1}, \ldots, p_{K}$ and let $P^{\perp}$ be the orthogonal complement of $P$ in $\mathbb{R}^{r}$.

We remark that the bound for $|\bar{x}|$ given by Lemma 10 is not in closed form. We could run an SDP tool to compute an upper bound for $s(r, \beta, \gamma)$ or possibly apply another result about twodistance spherical sets. However, if $1 \leq n \leq K-((1 / \alpha)+1) / 2$, we can modify Lemma 10 and derive explicit bounds, see Lemmas 1214 below.

Lemma 12. If $1<n<K-((1 / \alpha)+1) / 2$, then for any $x \in X(K, n),|\bar{x}| \leq r+1$.

Proof. By Proposition 4 (7), if $n<K-((1 / \alpha)+1) / 2$, then $\alpha<\ell(K, n)<1$. So both $\beta=$ $(\alpha-\ell(K, n)) /(1-\ell(K, n))$ and $\gamma=(-\alpha-\ell(K, n)) /(1-\ell(K, n))$ are negative. By Lemma 10 and (Barg and Yu, 2013, Theorem 3.2, last case), $|\bar{x}| \leq s(r, \beta, \gamma) \leq r+1$.

Lemma 13. If $n=K-((1 / \alpha)+1) / 2$, then for any $x \in X(K, n),|\bar{x}| \leq r-K+\left\lfloor 2 \alpha \frac{r-K}{1-\alpha}\right\rfloor$.

Proof. By Proposition 4 (7), if $n=K-((1 / \alpha)+1) / 2$, then $\ell(K, n)=\alpha$. So $\beta=(\alpha-\ell(K, n)) /(1-$ $\ell(K, n))=0$ and $\gamma=(-\alpha-\ell(K, n)) /(1-\ell(K, n))=-2 \alpha /(1-\alpha)$. By (Lemmens and Seidel, 1973, Theorem 4.1), $|\bar{x}| \leq r-K+\lfloor 2 \alpha(r-K) /(1-\alpha)\rfloor$.

Lemma 14. For any $x \in X(K, 1)$,

$$
|\bar{x}| \leq\left\{\begin{array}{ll}
r-K, & 1 \geq K-\frac{(1 / \alpha)+1}{2} \\
\frac{1-\alpha}{\ell(K, 1)-\alpha}, & 1<K-\frac{(1 / \alpha)+1}{2},
\end{array} \quad \text { where } 0<\alpha<1 .\right.
$$

Proof. Let $\bar{x}=\left\{x_{1}, \ldots, x_{s}\right\}$. By Corollary 1, we can assume that for each $i=1, \ldots, s$,

$$
x_{i}=h+c_{i}, \quad \text { where } h \in P \text { and } c_{i} \in P^{\perp},
$$

and for all $x \in \bar{x} \subset X(K, 1)$, there exist $K-1$ vectors among $p_{1}, \ldots, p_{K}$, say $p_{1}, \ldots, p_{K-1}$, such that for any $i=1, \ldots, K-1,\left\langle p_{i}, x\right\rangle=-\alpha$.

Assume there exist $x, \tilde{x} \in \bar{x} \subset X(K, 1)$ such that $\langle x, \tilde{x}\rangle=-\alpha$. Then the $K+1$ vectors $p_{1}, \ldots, p_{K-1}, x, \tilde{x} \in X$ have pairwise inner product $-\alpha$ and hence $K_{\alpha}(X) \geq K+1$, which contradicts to the hypothesis that $K_{\alpha}(X)=K$. So we must have $\langle x, \tilde{x}\rangle=\alpha$ for any $x, \tilde{x} \in \bar{x}$.

By the above argument, we have $G(\bar{x})=(1-\alpha) I+\alpha J$. By Proposition $4,\langle h, h\rangle=\ell(K, 1)$. Thus,

$$
G\left(c_{1}, \ldots, c_{s}\right)=G(\bar{x})-\ell(K, 1) \cdot J=(1-\alpha) I-(\ell(K, 1)-\alpha) J
$$

By Lemma 4, the above matrix has two eigenvalues

$$
\lambda_{1}=1-\ell(K, 1)+(s-1)(\alpha-\ell(K, 1)), \quad \lambda_{2}=1-\alpha .
$$

For the first case $1 \geq K-((1 / \alpha)+1) / 2$, by Proposition 4 (7), we have $\ell(K, 1) \leq \alpha<1$ in this case. Hence, it is seen from (17) that both eigenvalues $\lambda_{1}$ and $\lambda_{2}$ are strictly positive. So the matrix $G(\bar{x})-\ell(K, 1) \cdot J$ is full rank and the rank is $s$. Note $c_{1}, \ldots, c_{s} \in P^{\perp}$ and $P^{\perp}$ has dimension $r-K$. Thus, by Lemma1, we have $s \leq r-K$. 
Now we discuss the second case $1<K-((1 / \alpha)+1) / 2$. Note $G\left(c_{1}, \ldots, c_{s}\right)$ is positive semidefinite and hence all its eigenvalues should be non-negative. By checking $\lambda_{1}$, we have

$$
\lambda_{1}=1-\ell(K, 1)+(s-1)(\alpha-\ell(K, 1)) \geq 0 \Longleftrightarrow s \leq \frac{1-\alpha}{\ell(K, 1)-\alpha} .
$$

(We remark that by Proposition 4 (7), if $1<K-((1 / \alpha)+1) / 2$, then $\alpha<\ell(K, 1)<1$. So the above bound for $s$ is non-trivial.)

\section{Proof of Lemma 7}

The goal of this subsection is to prove Lemma 7 . Suppose we have an equiangular set $X$ in $\mathbb{R}^{r}$ with angle $1 / 5$ and we have $K=K_{1 / 5}(X)=4$. Fix a $K$-base $\left\{p_{1}, p_{2}, p_{3}, p_{4}\right\}$. Define $X(K, n)$ as in (11). Then the partition (12) becomes

$$
X=\left\{p_{1}, p_{2}, p_{3}, p_{4}\right\} \bigcup X(4,1) \bigcup X(4,2) .
$$

By Theorem [5, there are $\left(\begin{array}{l}4 \\ 1\end{array}\right)=4$ equivalence classes in $X(4,1)$, say $\bar{x}_{m}(m=1,2,3,4)$, with

$$
|X(4,1)|=\sum_{m=1}^{4}\left|\bar{x}_{m}\right| .
$$

and $\frac{1}{2}\left(\begin{array}{l}4 \\ 2\end{array}\right)=3$ equivalence classes in $X(4,2)$, say $\bar{x}_{m}(m=5,6,7)$ with

$$
|X(4,2)|=\sum_{m=5}^{7}\left|\bar{x}_{m}\right| .
$$

So by the partition in (18), we have

$$
|X|=4+|X(4,1)|+|X(4,2)|=4+\sum_{m=1}^{4}\left|\bar{x}_{m}\right|+\sum_{m=5}^{7}\left|\bar{x}_{m}\right| .
$$

Below, we discuss the upper bound for each $\left|\bar{x}_{m}\right|$ in Lemmas 16] and 17. After that, we give the proof of Lemma 7 .

Lemma 15. If a real $(s+1) \times(s+1)$-matrix $(s>2)$

$$
G=\left(\begin{array}{cccc}
a & \ldots & 0 & i_{1} \\
\vdots & \ddots & \vdots & \vdots \\
0 & \ldots & a & i_{s} \\
i_{1} & \ldots & i_{s} & b
\end{array}\right)
$$

is positive semidefinite, then $\sum_{k=1}^{s} i_{k}^{2} \leq a b$.

Proof. It is straightforward to compute that the characteristic polynomial of the matrix $G$ is

$$
(\lambda-a)^{s-2}\left(\lambda^{2}-(a+b) \lambda+a b-\sum_{k=1}^{s} i_{k}^{2}\right) .
$$

Because $G$ is positive semidefinite, we have all non-negative eigenvalues. Hence, the product of last two eigenvalues should be non-negative. That means $a b-\sum_{k=1}^{s} i_{k}^{2} \geq 0$. 
Lemma 16. If $x \in X(4,1)$ and there exists another vector $y \in X$ such that $y \notin \bar{x}$, then we have the statements below.

(1) If $y \in X(4,1)$, then $|\bar{x}| \leq 36$.

(2) If $y \in X(4,2)$, then $|\bar{x}| \leq 39$.

Proof. Since $x \in X(4,1)$, by the definition of $X(4,1)$ in (11), there is exactly 1 positive inner product or $4-1=3$ positive inner products among $\left\langle x, p_{i}\right\rangle, i=1,2,3,4$. Without loss of generality, we assume

$$
\left(\left\langle x, p_{1}\right\rangle,\left\langle x, p_{2}\right\rangle,\left\langle x, p_{3}\right\rangle,\left\langle x, p_{4}\right\rangle\right)^{\top}=\frac{1}{5}(-1,1,1,1)^{\top} .
$$

If it is in the other cases, one can derive the same conclusion because of symmetry.

Suppose $x=h+c$ where $h \in P$ and $c \in P^{\perp}$. By Proposition 4.

$$
h=a^{(1)} p_{1}+\cdots+a^{(4)} p_{4}=\frac{p_{2}+p_{3}+p_{4}}{3},
$$

where

$$
\left(a^{(1)}, a^{(2)}, a^{(3)}, a^{(4)}\right)=\alpha\left((1+\alpha) I_{4}-\alpha J_{4}\right)^{-1}(-1,1,1,1)^{\top}=(0,1 / 3,1 / 3,1 / 3) .
$$

Here, note $\left.\left((1+\alpha) I_{4}-\alpha J_{4}\right)^{-1}\right|_{\alpha=1 / 5}=(5 / 4) I_{4}+(5 / 12) J_{4}$, and by (6),

$$
\langle h, h\rangle=\ell(4,1)=\left.\frac{\alpha^{2}(4 \alpha n(n-K)+K(1+\alpha))}{(1+\alpha)(1-(K-1) \alpha)}\right|_{\alpha=1 / 5, n=1, K=4}=\frac{1}{5} .
$$

Assume

$$
\left(\left\langle y, p_{1}\right\rangle,\left\langle y, p_{2}\right\rangle,\left\langle y, p_{3}\right\rangle,\left\langle y, p_{4}\right\rangle\right)^{\top}=\frac{1}{5} \epsilon .
$$

Since $y \notin \bar{x}$, by Lemma 5 , $\epsilon$ can not be $\pm(-1,1,1,1)^{\top}$.

(1) If $y \in X(4,1)$, then $\epsilon$ can be one of the six vectors below

$$
\pm(1,-1,1,1)^{\top}, \pm(1,1,-1,1)^{\top}, \pm(1,1,1,-1)^{\top} .
$$

Suppose $y=g+d$ where $g \in P$ and $d \in P^{\perp}$. According to those above six possible $\epsilon$ 's, by Proposition $4 g$ will be respectively

$$
\pm \frac{p_{1}+p_{3}+p_{4}}{3}, \pm \frac{p_{1}+p_{2}+p_{4}}{3}, \pm \frac{p_{1}+p_{2}+p_{3}}{3} .
$$

However, remark that in all cases, we have

$$
\langle g, g\rangle=1 / 5 .
$$

Let $\bar{x}=\left\{x_{1}, \ldots, x_{s}\right\}$. By Corollary 1, we can assume that for each $i=1, \ldots, s$,

$$
x_{i}=h+c_{i}, \text { where } c_{i} \in P^{\perp} .
$$


Recall the proof of Lemma 14 that for any different $x_{i}, x_{j} \in \bar{x} \subset X(4,1)$, we have

$$
\left\langle x_{i}, x_{j}\right\rangle=1 / 5 \text {. }
$$

Thus, by (21), (23) and (24), the Gramian matrix of $c_{1}, \ldots, c_{s}, d$ is

$$
\begin{aligned}
& \left(\begin{array}{cccc}
\left\langle c_{1}, c_{1}\right\rangle & \ldots & \left\langle c_{1}, c_{s}\right\rangle & \left\langle c_{1}, d\right\rangle \\
\vdots & \ddots & \vdots & \vdots \\
\left\langle c_{s}, c_{1}\right\rangle & \ldots & \left\langle c_{s}, c_{s}\right\rangle & \left\langle c_{s}, d\right\rangle \\
\left\langle d, c_{1}\right\rangle & \ldots & \left\langle d, c_{s}\right\rangle & \langle d, d\rangle
\end{array}\right)=\left(\begin{array}{cccc}
\left\langle x_{1}, x_{1}\right\rangle & \ldots & \left\langle x_{1}, x_{s}\right\rangle & \left\langle x_{1}, y\right\rangle \\
\vdots & \ddots & \vdots & \vdots \\
\left\langle x_{s}, x_{1}\right\rangle & \ldots & \left\langle x_{s}, x_{s}\right\rangle & \left\langle x_{s}, y\right\rangle \\
\left\langle y, x_{1}\right\rangle & \ldots & \left\langle y, x_{s}\right\rangle & \langle y, y\rangle
\end{array}\right)-\left(\begin{array}{cccc}
\langle h, h\rangle & \ldots & \langle h, h\rangle & \langle h, g\rangle \\
\vdots & \ddots & \vdots & \vdots \\
\langle h, h\rangle & \ldots & \langle h, h\rangle & \langle h, g\rangle \\
\langle g, h\rangle & \ldots & \langle g, h\rangle & \langle g, g\rangle
\end{array}\right) \\
& =\left(\begin{array}{cccc}
1 & \cdots & 1 / 5 & \left\langle x_{1}, y\right\rangle \\
\vdots & \ddots & \vdots & \vdots \\
1 / 5 & \cdots & 1 & \left\langle x_{s}, y\right\rangle \\
\left\langle y, x_{1}\right\rangle & \cdots & \left\langle y, x_{s}\right\rangle & 1
\end{array}\right)-\left(\begin{array}{cccc}
1 / 5 & \cdots & 1 / 5 & \langle h, g\rangle \\
\vdots & \ddots & \vdots & \vdots \\
1 / 5 & \cdots & 1 / 5 & \langle h, g\rangle \\
\langle h, g\rangle & \cdots & \langle h, g\rangle & 1 / 5
\end{array}\right) \\
& =\left(\begin{array}{cccc}
4 / 5 & \cdots & 0 & \left\langle x_{1}, y\right\rangle-\langle h, g\rangle \\
\vdots & \ddots & \vdots & \vdots \\
0 & \cdots & 4 / 5 & \left\langle x_{s}, y\right\rangle-\langle h, g\rangle \\
\left\langle y, x_{1}\right\rangle-\langle h, g\rangle & \cdots & \left\langle y, x_{s}\right\rangle-\langle h, g\rangle & 4 / 5
\end{array}\right) .
\end{aligned}
$$

For $i=1, \ldots, s$, let $i_{k}=\left\langle x_{k}, y\right\rangle-\langle h, g\rangle \quad\left(=\left\langle y, x_{k}\right\rangle-\langle h, g\rangle\right)$. Here in order to apply Lemma 15. we assume $s>2$; otherwise, the conclusion $s \leq 36$ we want to prove will be naturally true. Then by Lemma 15, $\sum_{k=1}^{s} i_{k}^{2} \leq\left(\frac{4}{5}\right)^{2}$. Note also $\left\langle x_{k}, y\right\rangle$ is $\pm 1 / 5$ since $\bar{x} \cup\{y\} \subset X$ is equiangular. By (20), (22) and the fact that $\left\langle p_{i}, p_{j}\right\rangle=-1 / 5$ for any $i \neq j$, one can compute directly that $\langle h, g\rangle$ is $\pm 1 / 15$.

So

$$
\left|i_{k}\right|=\left|\left\langle x_{k}, y\right\rangle-\langle h, g\rangle\right| \geq(1 / 5)-(1 / 15)=(2 / 15) .
$$

Thus,

$$
s \cdot\left(\frac{2}{15}\right)^{2} \leq \sum_{k=1}^{s} i_{k}^{2} \leq\left(\frac{4}{5}\right)^{2} \Rightarrow s \leq \frac{(4 / 5)^{2}}{(2 / 15)^{2}}=36 .
$$

(2) If $y \in X(4,2)$, then $\epsilon$ has the six possibilities below

$$
\pm(1,1,-1,-1)^{\top}, \pm(1,-1,1,-1)^{\top}, \pm(1,-1,-1,1)^{\top} .
$$

According to these six $\epsilon$ 's, by Proposition 4 . $g$ will respectively be

$$
\pm \frac{p_{1}+p_{2}-p_{3}-p_{4}}{6}, \pm \frac{p_{1}-p_{2}+p_{3}-p_{4}}{6}, \pm \frac{p_{1}-p_{2}-p_{3}+p_{4}}{6} .
$$

However, in all cases, we have $\langle g, g\rangle=2 / 15$ and $\langle h, g\rangle= \pm 1 / 15$. Let $\bar{x}=\left\{x_{1}, \ldots, x_{s}\right\}$. By Corollary 1 we can assume that

$$
x_{i}=h+c_{i} \text { for each } i=1, \ldots, s, \text { where } c_{i} \in P^{\perp} .
$$

Similar to what we have done in the case (1), one can compute that the Gramian matrix of $c_{1}, \ldots, c_{s}, d$ is

$$
\left(\begin{array}{cccc}
\left\langle c_{1}, c_{1}\right\rangle & \ldots & \left\langle c_{1}, c_{s}\right\rangle & \left\langle c_{1}, d\right\rangle \\
\vdots & \ddots & \vdots & \vdots \\
\left\langle c_{s}, c_{1}\right\rangle & \ldots & \left\langle c_{s}, c_{s}\right\rangle & \left\langle c_{s}, d\right\rangle \\
\left\langle d, c_{1}\right\rangle & \ldots & \left\langle d, c_{s}\right\rangle & \langle d, d\rangle
\end{array}\right)=\left(\begin{array}{cccc}
4 / 5 & \ldots & 0 & \left\langle x_{1}, y\right\rangle-\langle h, g\rangle \\
\vdots & \ddots & \vdots & \vdots \\
0 & \ldots & 4 / 5 & \left\langle x_{s}, y\right\rangle-\langle h, g\rangle \\
\left\langle y, x_{1}\right\rangle-\langle h, g\rangle & \ldots & \left\langle y, x_{s}\right\rangle-\langle h, g\rangle & 13 / 15
\end{array}\right) .
$$


Again, for $i=1, \ldots, s$, let $i_{k}=\left\langle x_{k}, y\right\rangle-\langle h, g\rangle$. By Lemma 15, $\sum_{k=1}^{s} i_{k}^{2} \leq \frac{4}{5} \cdot \frac{13}{15}=\frac{52}{75}$. Note again $\left\langle x_{k}, y\right\rangle$ is $\pm 1 / 5$ and $\langle h, g\rangle$ is $\pm 1 / 15$. So

$$
\left|i_{k}\right|=\left|\left\langle x_{k}, y\right\rangle-\langle h, g\rangle\right| \geq(1 / 5)-(1 / 15)=(2 / 15) .
$$

Thus, we have

$$
s \cdot\left(\frac{2}{15}\right)^{2} \leq \sum_{k=1}^{s} i_{k}^{2} \leq \frac{52}{75} \quad \Rightarrow \quad s \leq \frac{52 / 75}{(2 / 15)^{2}}=39 .
$$

Lemma 17. If $x \in X(4,2)$, then

$$
|\bar{x}| \leq s(r, 1 / 13,-5 / 13) .
$$

Proof. We note that $K-(1+(1 / \alpha)) / 2=4-(1+5) / 2=1<n=2$. So we apply Theorem 6 (4) to conclude that $|\bar{x}| \leq s(r, \beta, \gamma)$, with $\ell(4,2)=2 / 15, \beta=(1 / 5-2 / 15) /(1-2 / 15)=1 / 13$, and $\gamma=(-1 / 5-2 / 15) /(1-2 / 15)=-5 / 13$.

Proof of Lemma 7, Based on Formula (19) and Lemma [16] we have 4 cases to consider.

(Case 1). If $X(4,2)=\emptyset$ and there is only one non-empty equivalence class in $X(4,1)$, say $\bar{x}_{1}$, then by Theorem $6(1),\left|\bar{x}_{1}\right| \leq r-4$. So by Formula (19),

$$
|X|=4+\left|\bar{x}_{1}\right| \leq 4+r-4=r .
$$

(Case 2). If $X(4,2)=\emptyset$ and there are at least two non-empty equivalence classes in $X(4,1)$, then by Lemma 16 (1), for each equivalence class $\bar{x}_{m}$, we have $\left|\bar{x}_{m}\right| \leq 36$. So by the formula (19),

$$
|X|=4+|X(4,1)|=4+\sum_{m=1}^{4}\left|\bar{x}_{m}\right| \leq 4+4 \times 36=148 .
$$

(Case 3). If $X(4,2) \neq \emptyset$ and if there is only one non-empty equivalence class in $X(4,1)$, say $\bar{x}_{1}$, then by Lemma $16(2),\left|\bar{x}_{1}\right| \leq 39$, and by Lemma 17 for each $\bar{x}_{m} \subset X(4,2)$, we have

$$
\left|\bar{x}_{m}\right| \leq s(r, 1 / 13,-5 / 13), \quad m=5,6,7 .
$$

So by Formula (19),

$$
|X|=4+|X(4,1)|+|X(4,2)|=4+\left|\bar{x}_{1}\right|+\sum_{m=5}^{7}\left|\bar{x}_{m}\right| \leq 43+3 \cdot s(r, 1 / 13,-5 / 13) .
$$

(Case 4). If $X(4,2) \neq \emptyset$ and if there are at least two non-empty equivalence classes in $X(4,2)$, then by Lemma 16 (1), for each equivalence class $\bar{x}_{m}$, we have $\left|\bar{x}_{m}\right| \leq 36$, and by Lemma 17 for each $\bar{x}_{m} \subset X(4,2)$, we have

$$
\left|\bar{x}_{m}\right| \leq s(r, 1 / 13,-5 / 13), \quad m=5,6,7 .
$$


So by Formula (19),

$$
\begin{aligned}
|X| & =4+|X(4,1)|+|X(4,2)|=4+\sum_{m=1}^{7}\left|\bar{x}_{m}\right| \\
& \leq 4+4 \times 36+3 \cdot s(r, 1 / 13,-5 / 13)=148+3 \cdot s(r, 1 / 13,-5 / 13) .
\end{aligned}
$$

Note also for any $r$, by Corollary $3, s(r, 1 / 13,-5 / 13) \geq r$. So among the above 4 cases, the maximum upper bound of $|X|$ is that in the last case $148+3 \cdot s(r, 1 / 13,-5 / 13)$.

\section{Proof of Lemma 8}

The goal of this subsection is to prove Lemma 8 . Suppose we have an equiangular set $X$ in $\mathbb{R}^{r}$ with angle $1 / 5$ and $K=K_{1 / 5}(X)=5$. Fix a $K$-base $\left\{p_{1}, p_{2}, p_{3}, p_{4}, p_{5}\right\}$. Define $X(K, n)$ as in (11). Then the partition in (12) becomes

$$
X=\left\{p_{1}, \ldots, p_{5}\right\} \bigcup X(5,1) \bigcup X(5,2) .
$$

Below, we discuss the upper bounds for $|X(5,1)|$ and $\left|\left\{p_{1}, \ldots, p_{5}\right\} \cup X(5,2)\right|$ in Lemma 18 and Lemma 19, respectively. After that, we give the proof of Lemma 8.

\section{Lemma 18.}

$$
|X(5,1)| \leq 15 .
$$

Proof. In this case, we have $\alpha=1 / 5, K=5$. By Theorem [6 (1), for any $x \in X(5,1)$,

$$
|\bar{x}| \leq \frac{1-\alpha}{\ell(K, 1)-\alpha}=\frac{1-1 / 5}{\ell(5,1)-1 / 5}=3 .
$$

By Theorem 5 there are $\left(\begin{array}{l}5 \\ 1\end{array}\right)=5$ equivalence classes $\bar{x}$ in $X(5,1)$. So $|X(5,1)| \leq 5 \times 3=15$.

\section{Lemma 19.}

$$
\left|\left\{p_{1}, \ldots, p_{5}\right\} \bigcup X(5,2)\right| \leq \begin{cases}275, & 23 \leq r \leq 185 \\ r+\lfloor(r-5) / 2\rfloor, & r \geq 185 .\end{cases}
$$

Proof. Let $p_{6}=-\sum_{i=1}^{5} p_{i}$. Note for each $j=1, \ldots, 5$,

$$
\left\langle p_{j}, p_{6}\right\rangle=\left\langle p_{j},-\sum_{i=1}^{5} p_{i}\right\rangle=-1-4 \times(-1 / 5)=-1 / 5 .
$$

Note also for any $x \in X(5,2)$, by the definition of $X(5,2)$ in (11), there are 2 or $5-2=3$ positive $1 / 5$ 's among $\left\langle x, p_{i}\right\rangle$ for $i=1 \ldots 5$. If there are 2 positive $1 / 5$ 's in these 5 inner products, then

$$
\left\langle x, p_{6}\right\rangle=\left\langle x,-\sum_{i=1}^{5} p_{i}\right\rangle=-2 \times(1 / 5)-3 \times(-1 / 5)=1 / 5,
$$


and if there are 3 positive $1 / 5$ 's in the 5 inner products, then

$$
\left\langle x, p_{6}\right\rangle=\left\langle x,-\sum_{i=1}^{5} p_{i}\right\rangle=-2 \times(-1 / 5)-3 \times(1 / 5)=-1 / 5 .
$$

Let

$$
Y=\left\{p_{6}\right\} \bigcup\left\{p_{1}, \ldots, p_{5}\right\} \bigcup X(5,2) .
$$

Then $Y$ is equiangular with angle $1 / 5$ in $\mathbb{R}^{r}$, and we have $K_{1 / 5}(Y)=6$ since

$$
G\left(p_{1}, \ldots, p_{6}\right)=(1+\alpha) I-\left.\alpha J\right|_{\alpha=1 / 5} .
$$

However, unlike in previous lemmas, $Y$ is not switching equivalent to a subset of $X$ as

$$
K_{1 / 5}(X)=5<6=K_{1 / 5}(Y) .
$$

Thus, neither $p_{6}$ nor $-p_{6}$ is in $X$. But it still holds by Theorem 4 that,

$$
|Y| \leq \begin{cases}276, & 23 \leq r \leq 185 \\ r+1+\lfloor(r-5) / 2\rfloor, & r \geq 185\end{cases}
$$

So we have

$$
\left|\left\{p_{1}, \ldots, p_{5}\right\} \bigcup X(5,2)\right|=|Y|-1 \leq \begin{cases}275, & 23 \leq r \leq 185 \\ r+\lfloor(r-5) / 2\rfloor, & r \geq 185\end{cases}
$$

Proof of Lemma 8. By Formula (25) and Lemmas 18, 19, we have

$$
|X|=|X(5,1)|+\left|\left\{p_{1}, \ldots, p_{5}\right\} \bigcup X(5,2)\right| \leq \begin{cases}290, & 23 \leq r \leq 185 \\ r+15+\lfloor(r-5) / 2\rfloor, & r \geq 185\end{cases}
$$

